\title{
THE DECOMPOSITION OF THE SPINOR BUNDLE OF GRASSMANN MANIFOLDS
}

\author{
FRANK KLINKER
}

\begin{abstract}
The decomposition of the spinor bundle of the spin Grassmann manifolds $G_{m, n}=S O(m+n) / S O(m) \times S O(n)$ into irreducible representations of $\mathfrak{s o}(m) \oplus \mathfrak{s o}(n)$ is presented. A universal construction is developed and the general statement is proven for $G_{2 k+1,3}, G_{2 k, 4}$, and $G_{2 k+1,5}$ for all $k$. The decomposition is used to discuss properties of the spectrum and the eigenspaces of the Dirac operator.
\end{abstract}

\section{INTRODUCTION}

The discussion of the spectrum of differential operators on spin symmetric and spin homogeneous spaces has been part of the literature for many years (see for example, [3], 25, 26, 227] or [22]). This topic brings together different aspects of geometry and representation theory, such as existence of spin structures on homogeneous spaces (e.g., 2] or [19]) and branching rules for representations (e.g., 29, 20, 14, [24, 12], 16], 15], [17, 44). In particular the work of Parthasarathy [25] yields an important theoretical tool to describe the spectrum and the eigenspaces of the Dirac operator of a spin symmetric space $G / K$. It may roughly be summarized as follows. In the first step, decompose the spinor bundle of $M$ in irreps of $K$. In the second step, list all $G$-representation which decomposition with respect to $K$ admits a summand from the list obtained in the first step. In 22 and 23 these tools have been noticed to be very powerful for the discussion of the first eigenvalue of the Dirac operator. Nevertheless the practical application of the theoretical tools contains many difficulties which are of course the reason why most authors, including ourselves, restrict to examples. If we consider symmetric spaces, in particular where both parts are of the same rank, the second step has mainly been solved for the classical groups in the literature cited above. For the first step we need branching rules for the isotropy group of $M$ with respect to the subgroup $K$. Therefore this step is more sophisticated in so far as the rank difference between $K$ and the isotropy group is big, in general. For example, this difference is $\operatorname{rk}(\mathfrak{s o}(4 k \ell))-\operatorname{rk}(\mathfrak{s o}(2 k) \oplus \mathfrak{s o}(2 \ell))=2 k \ell-k-\ell$ for the Grassmannian $G_{2 k, 2 \ell}$.

In this text we prove a formula for the decomposition of the spinor bundle of the spin Grassmannians $G_{m, n}=S O(m+n) / S O(m) \times S O(n)$ for $n \leqslant 5$. The construction also yields the decomposition of the spinor bundle of $G_{m, n}$ in the

Date: November 2, 2018.

2000 Mathematics Subject Classification. 17B10, 14M14, 53C27 .

Key words and phrases. spinor representation, branching rule, Grassmann manifold, Dirac operator. 
general case. See theorem 13 and theorem 19 as well as conjecture 12 and conjecture 18. This decomposition can be rephrased as branching of the spin representation of $\mathfrak{s o}(m n)$ with respect to the subalgebra $\mathfrak{s o}(m) \oplus \mathfrak{s o}(n)$ for which there are no general statement proven so far but only partial results for $n=1,2$ (see, e.g., [27]). Our proof needs the explicit construction of the weights cf. (24) and (25) but also a dimension analysis. For the case $(m, n)=(2 k, 4)$ this dimension analysis is reformulated in lemma 16. Here as well as in the odd dimensional cases with $n \leqslant 5$ we need closed expressions for sums over binomial terms which we prove in appendix A. Why and how the techniques provided in the proofs for $n \leqslant 5$ can be used for the general case and what the practical difficulties are, is explained in section 5 Nevertheless for fixed $(m, n)$ one can let any computer algebra system - for example, MAPLE - do the dimension calculation to tell one that the decomposition results are right in all these cases. But this is not the only evidence of the correctness of our general result. In section 4 we discuss some aspects of the spectrum and the eigenspaces of the Dirac operator on Grassmann manifolds. We compare our results of section 3 with the results of 22 and 23 . The perfect match also substantiates the statement on the decompositions (29) and (37) in the general case. As a further result we identify the smallest summand of the eigenspace to the first eigenvalue of the Dirac operator, see propositions 25] and 30 and conjectures 24 and 29, Small in this situation means with respect to the ordering which is induced by the ordering of weights. We show that this eigenspace is nondegenerate in the case $G_{4,4}$ (see example 28).

The text is organized as follows. In section 2 we recall the theoretical basis and explain the projection method at a well known simple example before we use this method to decompose the spinor bundle of the Grassmannians in section 3, In section 4 we turn to the discussion of the Dirac operator and its spectrum and end up with some concluding remarks in section 5 .

\section{Decomposing the Spinor Representation of $G / K$}

2.1. Symmetric spaces: The Parthasarathy formula. Consider a homogeneous spaces $M=G / K, K \subset G, \mathfrak{g}=\mathfrak{k} \oplus \mathfrak{p}$ together with its $G$-invariant Riemannian metric induced by the Killing form $b[1]$ Then

$$
\begin{aligned}
\mathfrak{p} & =\{v \in \mathfrak{g} \mid b(v, h)=0 \quad \forall h \in K\}, \\
{[\mathfrak{k}, \mathfrak{k}] } & \subset \mathfrak{k}, \quad[\mathfrak{k}, \mathfrak{p}] \subset \mathfrak{p}, \quad[\mathfrak{p}, \mathfrak{p}] \subset \mathfrak{k} \oplus \mathfrak{p} .
\end{aligned}
$$

Suppose $M$ is a symmetric space. Then, in particular, the bracket of $\mathfrak{p}$ with itself closes into $\mathfrak{k}$. Suppose $\mathfrak{k}$ and $\mathfrak{g}$ are of the same rank

Let $\zeta: K \rightarrow S O(\mathfrak{p})$ be the isotropy representation of $M . \zeta$ induces a representation $\zeta_{*}$ on Lie algebra level given by

$$
\zeta_{*}: \mathfrak{k} \rightarrow \mathfrak{s o}(\mathfrak{p}), \quad \zeta_{*}(h)(v)=\left.\left(a d_{h}^{\mathfrak{g}}\right)\right|_{\mathfrak{p}}(v)=\operatorname{proj}_{\mathfrak{p}}[h, v] .
$$

Remark 1. $M=G / K$ admits a $G$-invariant spin structure if and only if $\zeta$ lifts to $\tilde{\zeta}: K \rightarrow \operatorname{Spin}(\mathfrak{p})$.

\footnotetext{
${ }^{1}$ For $M$ isotropy irreducible we get an Einstein space with scalar curvature $s=\frac{\operatorname{dim} M}{2}$.

${ }^{2}$ This is true in almost all cases of symmetric spaces up to two series, see, for example, 11.
} 
Let $\left\{e_{i}\right\}$ be an orthonormal basis of $\mathfrak{p}$. Then $\zeta_{*}$ and $\tilde{\zeta}_{*}: \mathfrak{k} \rightarrow \mathfrak{s o}(\mathfrak{p})$ are connected via

$$
\tilde{\zeta}_{*}(h)=\frac{1}{4} \sum e_{i} \cdot \zeta_{*}(h)\left(e_{i}\right)
$$

where $\cdot$ denotes the Clifford multiplication in $C \ell(\mathfrak{p})$. We denote by $\gamma: \operatorname{Spin}(\mathfrak{p}) \rightarrow$ $\operatorname{End}(\Delta)$ the spinor representation and write $\rho:=\gamma \circ \tilde{\zeta}$. The following construction is due to 25] and has been used to calculate the eigenvalues of Dirac operators, see section 4

Let $S=G \times{ }_{\rho} \Delta$ be the spinor bundle of $M=G / K$, where $G$ is viewed as a principle bundle over $M$. S splits under the action of $\mathfrak{k}$ into certain subbundles which are labeled by an index set $\mathcal{W}_{0}$

$$
S=\bigoplus_{\sigma \in \mathcal{W}_{0}} S_{\sigma}
$$

Let $\Phi_{\mathfrak{g}}^{+}$and $\Phi_{\mathfrak{k}}^{+}$be the the sets of $\mathfrak{g}$-positive roots and $\mathfrak{k}$-positive roots, respectively. We define $\Phi_{\mathfrak{p}}^{+}:=\Phi_{\mathfrak{g}}^{+} \backslash \Phi_{\mathfrak{k}}^{+}$and

$$
\alpha_{\mathfrak{g}}:=\frac{1}{2} \sum_{\alpha \in \Phi_{\mathfrak{g}}^{+}} \alpha, \quad \alpha_{\mathfrak{k}}:=\frac{1}{2} \sum_{\alpha \in \Phi_{\mathfrak{k}}^{+}} \alpha .
$$

Let $\mathcal{W}$ be the Weyl group of $\mathfrak{g}$. Then $\mathcal{W}_{0}$ is given by

$$
\mathcal{W}_{0}=\left\{\sigma \in \mathcal{W} \mid \Phi_{\mathfrak{k}}^{+} \subset \sigma \Phi_{\mathfrak{g}}^{+}\right\}
$$

The spinor representation $\rho$ decomposes as $\rho=\sum_{\sigma \in \mathcal{W}_{0}} \rho_{\sigma}$, where

$$
\beta_{\sigma}=\sigma \alpha_{\mathfrak{g}}-\alpha_{\mathfrak{k}}=\frac{1}{2} \sum_{\alpha \in \sigma \Phi_{\mathfrak{p}}^{+}} \alpha
$$

is the highest weight of the irreducible representation $\rho_{\sigma}$ of $K$. The latter appears with multiplicity one in the sum. The corresponding representation space $S_{\sigma}$ is of dimension

$$
\operatorname{dim} S_{\sigma}=\sum_{\alpha \in \Phi_{\mathfrak{k}}^{+}} \frac{\left\langle\beta_{\sigma}+\alpha_{\mathfrak{k}}, \alpha\right\rangle}{\langle\alpha, \alpha\rangle}=\sum_{\alpha \in \Phi_{\mathfrak{k}}^{+}} \frac{\left\langle\sigma \alpha_{\mathfrak{g}}, \alpha\right\rangle}{\langle\alpha, \alpha\rangle} .
$$

Example 2. We consider $\mathfrak{g}=\mathfrak{s o}(2 n+1) \supset \mathfrak{s o}(2 n)=\mathfrak{k}$. The embedding is due to the usual $(\mathbf{2 n}+\mathbf{1})=\mathbf{2 n} \oplus \mathbf{1}$ splitting. The roots of $\mathfrak{s o}(2 n+1)$ are $\left\{ \pm e_{k}, \pm e_{i} \pm\right.$ $\left.e_{j}\right\}_{1 \leqslant k \leqslant n, 1 \leqslant i<j \leqslant n}$ and those of $\mathfrak{s o}(2 n)$ are given by the subset $\left\{ \pm e_{i} \pm e_{j}\right\}_{1 \leqslant i<j \leqslant n}$. The positive roots are $\Phi_{\mathfrak{s o}(2 n+1)}^{+}=\left\{e_{k}, e_{i} \pm e_{j}\right\}_{1 \leqslant k \leqslant n, 1 \leqslant i<j \leqslant n}$ and $\Phi_{\mathfrak{s o}(2 n)}^{+}=\left\{e_{i} \pm\right.$ $\left.e_{j}\right\}_{1 \leqslant i<j \leqslant n}$ respectively. So $\Phi_{\mathfrak{p}}^{+}=\left\{e_{k}\right\}_{1 \leqslant k \leqslant n}$. In particular $\alpha_{\mathfrak{s o}(2 n+1)}=\sum_{i=1}^{n}(n-$ $\left.i+\frac{1}{2}\right) e_{i}$ and $\alpha_{\mathfrak{s o}(2 n)}=\sum_{i=1}^{n}(n-i) e_{i}$. Beside the identity, the only Weyl reflection, $\sigma$, which obeys $\sigma \Phi_{\mathfrak{s o}(2 n+1)}^{+} \supset \Phi_{\mathfrak{s o}(2 n)}^{+}$is the one associated to the root $e_{n}$. This yields $\sigma \Phi_{\mathfrak{p}}^{+}=\left\{e_{k},-e_{n} \mid k=0, \ldots n-1\right\}$. Therefore, the spinor representation decomposes into two summands associated to the two highest weights

$$
\frac{1}{2}\left(e_{1}+\cdots e_{n-1} \pm e_{n}\right)
$$

as expected.

A generalization of the above construction by Parthasarathy is given by the following nice observation, see [6] or [9]. 
Proposition 3. Let $M=G / K$ be a symmetric space and $\mathfrak{g}$, $\mathfrak{k}$ semisimple with rank difference $d=\operatorname{rk}(\mathfrak{g})-\operatorname{rk}(\mathfrak{k})$. Let $\Pi$ be the set of highest weights of irreducible representations of $\mathfrak{k}$ which appear in the decomposition of the spinor representation of $M$. Then

$$
S=2^{\left[\frac{d}{2}\right]} \sum_{\beta \in \Pi} V_{\beta} .
$$

Example 4. Consider $M=G \times G / G$ with $\operatorname{dim} G=\operatorname{dim} M=n, \operatorname{rk}(G)=k$. In this case the isotropy representation is the adjoint action so that $V_{\mathfrak{s o}(n)}=a d_{\mathfrak{g}}$. The rank difference is exactly the $\operatorname{rank}$ of $G$ and the spinor representation is

$$
S=2^{\left[\frac{k}{2}\right]} V_{\alpha_{\mathfrak{g}}},
$$

with $\alpha_{\mathfrak{g}}$ from (44) - see [14.

2.2. General homogeneous spaces: the explicit construction. The method cf. Parthasarathy (6) does only work for the decomposition of the spinor bundle of symmetric spaces. If we want to deal with general homogeneous spaces we have to calculate the decomposition directly. Even in the situation of section 2.1 it is sometimes easy to do so, because we have to compare the weights of the respective algebras in a common base anyway.

We consider the homogeneous space $M=G / K$ with faithful isotropy representation $K \rightarrow S O(\mathfrak{p})$, where $\mathfrak{g}=\mathfrak{k} \oplus \mathfrak{p}$ is the decomposition of the associated Lie algebras. We suppose $M$ to be spin such that the isotropy representation $\zeta_{*}: \mathfrak{k} \rightarrow \mathfrak{s o}(\mathfrak{p})$ gives rise to the spinor representation $\rho: K \rightarrow \operatorname{Spin}(\mathfrak{p}) \subset \operatorname{End}(\Delta)$.

The construction: We decompose $a d_{\mathfrak{g}}$ with respect to $\mathfrak{k}$ and get

$$
a d_{\mathfrak{g}}=a d_{\mathfrak{k}} \oplus \bigoplus_{\alpha} V_{\alpha},
$$

where $\oplus_{\alpha} V_{\alpha}$ is the decomposition of $\zeta_{*}=\left.a d^{\mathfrak{g}}\right|_{\mathfrak{k}, \mathfrak{p}}$. From the construction we see that this coincides with the vector representation of $\mathfrak{s o}(\mathfrak{p})$ :

$$
\text { vector representation of } \mathfrak{s o}(\mathfrak{p}) \cong \bigoplus_{\alpha} V_{\alpha} \text {. }
$$

This information encodes the inclusion due to the following observation, see 31, sec. 7.6]

Proposition 5. Let $\iota: \mathfrak{h} \rightarrow \mathfrak{g}$ be an injective homomorphism of Lie algebras with $\mathfrak{h}$ semisimple. Consider the irreducible vector representation of $\mathfrak{g}$. Then the knowledge of its decomposition into irreducible representations with respect to $\mathfrak{h}$ yields the knowledge of the decomposition of any irreducible representation of $\mathfrak{g}$ with respect to $\mathfrak{h}$.

This proposition is related to the notion of plethyms and may be formulated as follows. Let $\lambda_{\mathfrak{g}}$ be the vector representation of $\mathfrak{g}$, then

$$
\lambda_{\mathfrak{g}} \searrow \sum_{i} \lambda_{\mathfrak{h}}^{i} \Rightarrow \mu_{\mathfrak{g}} \searrow \sum_{j} \mu_{\mathfrak{h}}^{j}=\sum_{i} \lambda_{\mathfrak{h}}^{i} \bar{\otimes} \mu_{\mathfrak{g}}
$$

where $\bar{\otimes}$ denotes the plethym. For a review on plethyms see, for example, [18] or 4. This yields that we are theoretically able to calculate the decomposition of the spinor representation of each homogeneous space. Nevertheless the decomposition 
contains many practical difficulties. We will illustrate the explicit construction at a well known example of low rank.

2.3. Example: Berger space $S O(5) / S O(3)$. Let $M$ be the seven dimensional Berger space. This is the homogeneous space $S O(5) / S O(3)$, where the subgroup is characterized as follows. We consider the embedding of $\mathfrak{s o}(3)$ in $\mathfrak{s o}(5)$ such that the five dimensional vector representation of $\mathfrak{s o}(5)$ stays irreducible.

We recall the weights of the five and four dimensional irreducible representations of $\mathfrak{s o}(5)$ and $\mathfrak{s o}(3)$ and the spaces associated to these representations.

\begin{tabular}{c|c|c|c|c} 
dim of & \multicolumn{2}{|c|}{$\mathfrak{s o}(5)$} & \multicolumn{2}{|c}{$\mathfrak{s o}(3)$} \\
Rep. & highest weight & space & highest weight & space \\
\hline $\mathbf{5}$ & $(1,0)=e_{1}$ & $\mathbb{R}^{5}$ & $(4)=4 \lambda$ & $S_{0}^{2}\left(\mathbb{R}^{3}\right)$ \\
\hline $\mathbf{4}$ & $\left(\frac{1}{2}, \frac{1}{2}\right)=\frac{1}{2}\left(e_{1}+e_{2}\right)$ & $S_{\frac{1}{2}}$ & $(3)=3 \lambda$ & $S_{\frac{3}{2}}$
\end{tabular}

Here we write $\lambda$ for the highest weight of the vector representation of $\mathfrak{s o}(3)$ so that the weights of $\mathfrak{s o}(3)$-representations are given by $k \lambda$.

The root system of $\mathfrak{s o}(5)$ is given by $\left\{ \pm e_{1}, \pm e_{2}, \pm e_{1} \pm e_{2}\right\}$.

To give $\lambda$ in terms of $\left\{e_{1}, e_{2}\right\}$ we use that the weight diagram of 5 with respect to $\mathfrak{s o}(5)$ projects onto the corresponding weight diagram with respect to $\mathfrak{s o}(3)$. The weight diagram of $\mathbf{5}$ with respect to $\mathfrak{s o}(5)$ is $\left\{ \pm e_{1}, \pm e_{2}, 0\right\}$. Up to symmetries we have

$$
\begin{aligned}
& \operatorname{proj}_{\operatorname{span}\{\lambda\}}\left(e_{1}\right)=4 \lambda, \\
& \operatorname{proj}_{\text {span }\{\lambda\}}\left(e_{2}\right)=2 \lambda,
\end{aligned}
$$

or

$$
\begin{array}{ccc}
\left\langle e_{1}, \lambda\right\rangle \frac{\lambda}{|\lambda|^{2}}=4 \lambda \quad & \wedge \quad\left\langle e_{2}, \lambda\right\rangle \frac{\lambda}{|\lambda|^{2}}=2 \lambda, \\
\lambda_{1}=4|\lambda|^{2} & \wedge \quad \lambda_{2}=2|\lambda|^{2} .
\end{array}
$$

From the last line we get $|\lambda|^{2}=\frac{1}{20}$ and so

$$
\lambda=\frac{1}{10}\left(2 e_{1}+e_{2}\right) .
$$

Remark 6 . The 4 of $\mathfrak{s o}(5)$ stays irreducible as well. Its weight diagram is given by $\left\{ \pm e_{1} \pm e_{2}\right\}$ and we have $\operatorname{proj}_{\text {span }\{\lambda\}}\left(\frac{1}{2}\left(e_{1} \pm e_{2}\right)\right)=(2 \pm 1) \lambda$.

To decompose the adjoint of $\mathfrak{s o}(5)$ with respect to $\mathfrak{s o}(3)$ we need the projection 3 of the weight diagram on $\operatorname{span}\{\lambda\}$ :

$$
\begin{aligned}
\operatorname{proj}_{\text {span }\{\lambda\}}\left(e_{1}+e_{2}\right) & =6 \lambda, \\
\operatorname{proj}_{\text {span }\{\lambda\}}\left(e_{1}\right) & =4 \lambda, \\
\operatorname{proj}_{\text {span }\{\lambda\}}\left(e_{2}\right) & =2 \lambda, \\
\operatorname{proj}_{\text {span }\{\lambda\}}\left(e_{1}-e_{2}\right) & =2 \lambda .
\end{aligned}
$$

\footnotetext{
${ }^{3}$ We omit the projection of the origin, because its multiplicity stays the same.
} 
We see that the image contains two diagrams to the highest weight $6 \lambda$ and $2 \lambda$, respectively, where $2 \lambda$ is the adjoint of $\mathfrak{s o}(3)$ and $6 \lambda$ the seven dimensional irreducible representation. Therefore

$$
\mathfrak{s o}(5)=\mathfrak{s o}(3) \oplus \mathbf{7} .
$$

The isotropy representation yields the embedding $\mathfrak{s o}(3) \hookrightarrow \mathfrak{s o}(7)$ and the calculations above show that this remains irreducible with respect to $\mathfrak{s o}(3)$.

To get the decomposition of the spinor representation, we need the weight diagram of $\mathfrak{s o}(3)$ as subset of the weight lattice of $\mathfrak{s o}(7)$. Therefore we turn to a three dimensional picture and write the roots of $\mathfrak{s o}(7)$ as $\left\{ \pm e_{1}, \pm e_{2}, \pm e_{3}, \pm e_{1} \pm e_{2}, \pm e_{1} \pm\right.$ $\left.e_{3}, \pm e_{2} \pm e_{3}\right\}$ such that the weight lattice of $\mathbf{7}$ is given by $\left\{ \pm e_{1}, \pm e_{2}, \pm e_{3}, 0\right\}$. As before we denote the highest weight of the vector representation of $\mathfrak{s o}(3)$ as $\lambda$ so that the seven dimensional representation is given by $6 \lambda$. We get

$$
\begin{aligned}
& \operatorname{proj}_{\text {span }\{\lambda\}}\left(e_{1}\right)=6 \lambda, \\
& \operatorname{proj}_{\text {span }\{\lambda\}}\left(e_{2}\right)=4 \lambda, \\
& \operatorname{proj}_{\text {span }\{\lambda\}}\left(e_{3}\right)=2 \lambda,
\end{aligned}
$$

Or

$$
\lambda=\frac{1}{56}(6,4,2) .
$$

The spin representation is eight dimensional and the weights are given by $\left\{\frac{1}{2}\left( \pm e_{1} \pm\right.\right.$ $\left.\left.e_{2} \pm e_{3}\right)\right\}$. The projection on the weight lattice of $\mathfrak{s o}(3)$ is

$$
\operatorname{proj}_{\text {span }\{\lambda\}}\left(\left\{\frac{1}{2}\left( \pm e_{1} \pm e_{2} \pm e_{3}\right)\right\}\right)=\{ \pm 6 \lambda, \pm 4 \lambda, \pm 2 \lambda, 0 \lambda\} \cup\{0 \lambda\},
$$

which yields

Proposition 7. The spinor representation of $M=S O(5) / S O(3)$ split into the (6) and (0) of $\mathfrak{s o}(3)$ :

$$
8=7 \oplus 1
$$

In particular the Berger space is a nearly parallel $G_{2}$-Einstein manifold, in particular it admits a connection which annihilates one spinor (see [1] and [7]).

\section{The SPINOR BUndLe of GRASSMANn MANifoldS}

We recall the following observation (see 2 or 27 ).

Proposition 8. The Grassmannian $G_{m, n}=S O(n+m) / S O(n) \times S O(m)$ is spin if and only if $m=1$ or $n=1$ or $m+n$ even.

In particular if the Grassmannian is not of type $G_{n, n-1}$ all even and odd dimensional spin Grassmanianns are of the form $G_{2 k, 2 \ell}$ and $G_{2 k+1,2 \ell+1}$, respectively. Therefore we divide this section into two parts dedicated to the even and odd dimensional spin Grassmannians, respectively. 
3.1. The even dimensional case. We consider the even dimensional spin Grassmannian $G_{2 k, 2 \ell}=S O(2(k+\ell)) /(S O(2 k) \times S O(2 \ell))$. Our goal is to decompose the spinor representation of $G_{2 k, 2 \ell}$ with respect to $\mathfrak{s o}(2 k) \oplus \mathfrak{s o}(2 \ell)$. We consider $\ell \leqslant k$ and restrict ourselves to $\ell \neq 1$ because this case is treated in detail in [27. We have

$$
\mathfrak{s o}(2(k+\ell))=\mathfrak{s o}(2 k) \oplus \mathfrak{s o}(2 \ell) \oplus(\mathbf{2} \mathbf{k} \otimes \mathbf{2} \ell) .
$$

This yields that the isotropy representation $\mathfrak{s o}(2 k) \oplus \mathfrak{s o}(2 \ell) \hookrightarrow \mathfrak{s o}(4 k \ell)$ is the standard embedding. This means that the vector representation of $\mathfrak{s o}(4 k \ell)$ decomposes as

$$
4 \mathrm{k} \ell=2 \mathrm{k} \otimes 2 \ell .
$$

Moreover we know that the adjoint representation decomposes as follows

$$
\mathfrak{s o}(4 k \ell)=\mathfrak{s o}(2 k) \oplus \mathfrak{s o}(2 \ell) \oplus\left(\mathfrak{s o}(2 k) \otimes S_{0}^{2}\left(\mathbb{R}^{2 \ell}\right)\right) \oplus\left(S_{0}^{2}\left(\mathbb{R}^{2 k}\right) \otimes \mathfrak{s o}(2 \ell)\right) .
$$

We construct a basis of the Cartan algebra of $\mathfrak{s o}(4 k \ell)$ such that we recover the Cartan basis of $\mathfrak{s o}(2 k)$ and $\mathfrak{s o}(2 \ell)$. We denote the Cartan basis of $\mathfrak{s o}(2 k)$ and $\mathfrak{s o}(2 \ell)$ by $\left\{K_{i}\right\}_{1 \leqslant i \leqslant k}$ and $\left\{L_{i}\right\}_{1 \leqslant i \leqslant \ell}$, respectively. The associated decompositions into two dimensional subspaces are $\mathbb{R}^{2 k}=V_{1} \oplus \cdots \oplus V_{k}$ and $\mathbb{R}^{2 \ell}=W_{1} \oplus \cdots \oplus W_{\ell}$ with $V_{i}=\operatorname{span}\left\{v_{1}^{i}, v_{2}^{i}\right\}, W_{j}=\operatorname{span}\left\{w_{1}^{j}, w_{2}^{j}\right\}$, i.e., $K_{i}\left(v_{1}^{j}\right)=\delta_{i}^{j} v_{2}^{i}, K_{i}\left(v_{2}^{j}\right)=-\delta_{i}^{j} v_{1}^{i}$ and similar for $L_{i}$.

We write $V_{i} \otimes W_{j}=E_{i j} \oplus F_{i j}$ with $E_{i j}=\operatorname{span}\left\{e_{+}^{i j}, e_{-}^{i j}\right\}, F_{i j}=\operatorname{span}\left\{f_{+}^{i j}, f_{-}^{i j}\right\}$ and

$$
\begin{aligned}
e_{+}^{i j}=\frac{1}{\sqrt{2}}\left(v_{1}^{i} \otimes w_{1}^{j}+v_{2}^{i} \otimes w_{2}^{j}\right), & e_{-}^{i j}=\frac{1}{\sqrt{2}}\left(v_{2}^{i} \otimes w_{1}^{j}-v_{1}^{i} \otimes w_{2}^{j}\right), \\
f_{+}^{i j}=\frac{1}{\sqrt{2}}\left(v_{1}^{i} \otimes w_{2}^{j}+v_{2}^{i} \otimes w_{1}^{j}\right), & f_{-}^{i j}=\frac{1}{\sqrt{2}}\left(v_{1}^{i} \otimes w_{1}^{j}-v_{2}^{i} \otimes w_{2}^{j}\right) .
\end{aligned}
$$

We define $\left\{N_{i j}^{e}, N_{i j}^{f}\right\}_{1 \leqslant i \leqslant k, 1 \leqslant j \leqslant \ell}$ by

$$
\begin{aligned}
& N_{i j}^{e}\left(e_{ \pm}^{i^{\prime} j^{\prime}}\right)= \pm \delta_{i}^{i^{\prime}} \delta_{j}^{j^{\prime}} e_{\overline{+}}^{i j}, \\
& N_{i j}^{f}\left(f_{ \pm}^{i^{\prime} j^{\prime}}\right)= \pm \delta_{i}^{i^{\prime}} \delta_{j}^{j^{\prime}} f_{\bar{\mp}}^{i j} .
\end{aligned}
$$

This is the Cartan basis with associated decomposition

$$
\mathbb{R}^{4 k \ell}=\bigoplus_{i, j}\left(V_{i} \otimes W_{j}\right)=\bigoplus_{i, j} E_{i j} \oplus \bigoplus_{i, j} F_{i j}
$$

We have

$$
\begin{aligned}
& K_{i}\left(e_{ \pm}^{i^{\prime} j}\right)= \pm \delta_{i}^{i^{\prime}} e_{\mp}^{i j}, K_{i}\left(f_{ \pm}^{i^{\prime} j}\right)=\mp \delta_{i}^{i^{\prime}} f_{\mp}^{i j}, \\
& L_{j}\left(f_{ \pm}^{i j^{\prime}}\right)=\mp \delta_{j}^{j^{\prime}} f_{\bar{\mp}}^{i j}, L_{j}\left(e_{ \pm}^{i j^{\prime}}\right)=\mp \delta_{j}^{j^{\prime}} e_{\bar{\mp}}^{i j},
\end{aligned}
$$

for all $i=1, \ldots, k$ and $j=1, \ldots, \ell$, such that

$$
K_{i}=\sum_{j=1}^{\ell}\left(N_{i j}^{e}-N_{i j}^{f}\right) \text { and } L_{j}=-\sum_{i=1}^{k}\left(N_{i j}^{e}+N_{i j}^{f}\right) .
$$

We write $\epsilon_{i j}^{x}=\left(N_{i j}^{x}\right)^{*}$ such that the roots of $\mathfrak{s o}(4 k \ell)$ are given by

$$
\left\{ \pm \epsilon_{i j}^{x} \pm \epsilon_{i^{\prime} j^{\prime}}^{y} \mid x, y \in\{e, f\} ; 1 \leqslant i, i^{\prime} \leqslant k ; 1 \leqslant j, j^{\prime} \leqslant \ell\right\} .
$$


The roots which form the subalgebra $\mathfrak{s o}(2 k) \oplus \mathfrak{s o}(2 \ell)$ are given by $\left\{ \pm K_{i}^{*} \pm K_{i^{\prime}}^{*}\right\}$ and $\left\{ \pm L_{j}^{*} \pm L_{j^{\prime}}^{*}\right\}$ or

$$
\begin{aligned}
\left\{\sum_{j=1}^{\ell}\left( \pm\left(\epsilon_{i j}^{e}-\epsilon_{i j}^{f}\right) \pm\left(\epsilon_{i^{\prime} j}^{e}-\epsilon_{i^{\prime} j}^{f}\right)\right) \mid 1 \leqslant i, i^{\prime} \leqslant k\right\} \\
\cup\left\{\sum_{i=1}^{k}\left( \pm\left(\epsilon_{i j}^{f}+\epsilon_{i j}^{e}\right) \pm\left(\epsilon_{i j^{\prime}}^{f}+\epsilon_{i j^{\prime}}^{e}\right)\right) \mid 1 \leqslant j, j^{\prime} \leqslant \ell\right\} .
\end{aligned}
$$

To get the decomposition of the spinor representation of $\mathfrak{s o}(4 k \ell)$ with respect to $\mathfrak{s o}(2 k) \oplus \mathfrak{s o}(2 \ell)$ we project the diagram $\frac{1}{2}( \pm 1, \ldots, \pm 1)=\frac{1}{2} \sum_{x, i, j}\left( \pm \epsilon_{i j}^{x}\right)$ onto the subspace spanned by (23) and expand the result with respect to $\left\{K_{i}^{*}, L_{j}^{*}\right\}$. Explicitly this is done by writing

$$
\left[\begin{array}{c}
K_{i}^{*} \\
L_{j}^{*}
\end{array}\right]=A\left[\begin{array}{c}
\epsilon_{i j}^{e} \\
\epsilon_{i j}^{f}
\end{array}\right]
$$

If we choose $\epsilon_{i j}^{x}=\left(\epsilon_{11}^{x}, \ldots, \epsilon_{1 \ell}^{x}, \ldots, \epsilon_{k 1}^{x}, \ldots, \epsilon_{k \ell}^{x}\right)$ the $[(k+\ell) \times 2 k \ell]$-matrix $A$ is given by the following rows:

$$
\begin{aligned}
& A_{i}=(\overbrace{0, \ldots, 0}^{(i-1) \ell}, \overbrace{1 \ldots, 1}^{\ell}, \overbrace{0, \ldots, 0}^{(k-i) \ell}, \overbrace{0, \ldots, 0}^{(i-1) \ell}, \overbrace{-1 \ldots,-1}^{\ell}, \overbrace{0, \ldots, 0}^{(k-i) \ell}), \quad 1 \leqslant i \leqslant k,
\end{aligned}
$$

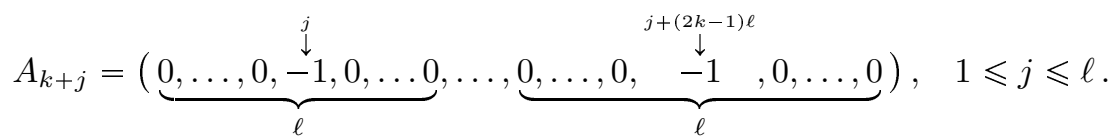

The projected diagram is read from

$$
\frac{1}{2} A( \pm 1, \ldots, \pm 1)^{T}
$$

and we get the first observation.

Remark 9. All the images of the set $\left\{\frac{1}{2}( \pm 1, \ldots, \pm 1)^{T}\right\}$ by the map $A$ consist of weights with integer entries.

To illustrate this procedure we will examine the examples $k=\ell=2, k=\ell=3$, and $k=4, \ell=2$ before we state the general result.

$\underline{k=\ell=2:}$ The matrix $A$ with $\left(4 K_{i}^{*}, 4 L_{j}^{*}\right)^{T}=A\left(\epsilon_{i j}^{e}, \epsilon_{i j}^{f}\right)^{T}$ is explicitly given by

$$
A=\left[\begin{array}{cccccccc}
1 & 1 & & & -1 & -1 & & \\
& & 1 & 1 & & & -1 & -1 \\
-1 & & -1 & & -1 & & -1 & \\
& -1 & & -1 & & -1 & & -1
\end{array}\right]
$$

\footnotetext{
${ }^{4}$ The diagram splits into two diagrams which correspond to the positive, respectively negative spinor representations depending on whether the number of minus signs is even, respectively odd.
} 
To get the image of a vector $\vec{x} \in \operatorname{span}\left\{\epsilon_{i j}^{x}\right\}$ under the projection we need $A \vec{x}$. For $\vec{x}$ contained in the spinor diagram the images are, for example,

$$
\begin{aligned}
& \frac{1}{2}(-1, \mp 1,-1, \mp 1,-1, \mp 1,-1, \mp 1) \longmapsto((0,0),(2, \pm 2)), \\
& \frac{1}{2}(+1,+1, \pm 1, \pm 1,-1,-1, \mp 1, \mp 1) \longmapsto((2, \pm 2),(0,0)), \\
& \frac{1}{2}(-1,+1, \mp 1, \pm 1,-1,-1, \mp 1, \mp 1) \longmapsto((1, \pm 1),(2,0)), \\
& \frac{1}{2}(+1,+1,-1, \mp 1,-1,-1,-1, \mp 1) \longmapsto((2,0),(1, \pm 1)), \\
& \frac{1}{2}(-1,+1,-1, \mp 1,-1,-1,-1, \mp 1) \longmapsto((1,0),(2, \pm 1)), \\
& \frac{1}{2}(+1,+1,-1, \pm 1,-1,-1,-1, \mp 1) \longmapsto((2, \pm 1),(1,0)) .
\end{aligned}
$$

$(2, \pm 2)$ may be identified with the subset of trace-free 4 -tensors on $\mathbb{R}^{4}$ with symmetry of the Young diagram $\square$. $(2, \pm 1)$ is given by the subset of trace-free 3 -tensors with symmetry $\boxminus$. In both cases \pm indicates the eigenspaces of the symmetry of the tensors, which is induced by the self duality of two forms in dimension 4 .

In table 1 we list the representation spaces, associated Young diagrams, and add the dimension as well as the further decomposition with respect to $\mathfrak{s u}(2) \oplus \mathfrak{s u}(2)$. The notation has been taken from 21] and contains the highest weights of the two factors, e.g., $(3 \mid 1)=\mathbf{4} \otimes \mathbf{2}$.

TABLE 1. Representations of $\mathfrak{s o}(4)$

\begin{tabular}{c|c|c|c} 
Rep. of $\mathfrak{s o}(4)$ & Symmetry & Dec. wrt. $\mathfrak{s u}(2) \oplus \mathfrak{s u}(2)$ & Dimension \\
\hline$(00)$ & $\cdot$ & $(0 \mid 0)$ & 1 \\
$(10)$ & $\square$ & $(1 \mid 1)$ & 4 \\
$\left(11^{ \pm}\right)$ & $\boxminus$ & $(2 \mid 0)$ and $(0 \mid 2)$ & $2 \cdot 3$ \\
$(20)$ & $\square$ & $(2 \mid 2)$ & 9 \\
$\left(22^{ \pm}\right)$ & $\square_{0}$ & $(4 \mid 0)$ and $(0 \mid 4)$ & $2 \cdot 5$ \\
$\left(21^{ \pm}\right)$ & $\square_{0}$ & $(3 \mid 1)$ and $(1 \mid 3)$ & $2 \cdot 8$
\end{tabular}

So the decomposition of the spinor representation into (reducible) representation spaces with respect to $\mathfrak{s o}(4) \oplus \mathfrak{s o}(4)$ is

$$
\begin{aligned}
& S^{+}=(1 \otimes 10) \oplus(10 \otimes 1) \oplus(6 \otimes 9) \oplus(9 \otimes 6), \\
& S^{-}=(4 \otimes 16) \oplus(16 \otimes 4) .
\end{aligned}
$$

and the irreducible decomposition - or equivalently the decomposition with respect to $\mathfrak{s u}(2) \oplus \mathfrak{s u}(2) \oplus \mathfrak{s u}(2) \oplus \mathfrak{s u}(2)-$ is

$$
\begin{aligned}
S^{+}= & (0|0| 0 \mid 4) \oplus(0|0| 4 \mid 0) \oplus(0|4| 0 \mid 0) \oplus(4|0| 0 \mid 0) \\
& \oplus(2|2| 2 \mid 0) \oplus(2|2| 0 \mid 2) \oplus(2|0| 2 \mid 2) \oplus(0|2| 2 \mid 2), \\
S^{-}= & (1|1| 1 \mid 3) \oplus(1|1| 3 \mid 1) \oplus(1|3| 1 \mid 1) \oplus(3|1| 1 \mid 1) .
\end{aligned}
$$


$\underline{k=4, \ell=2:}$ The matrix with $\left(4 K_{i}^{*}, 8 L_{i}^{*}\right)^{T}=A\left(\epsilon_{i j}^{e}, \epsilon_{i j}^{f}\right)^{T}$ is explicitly given by

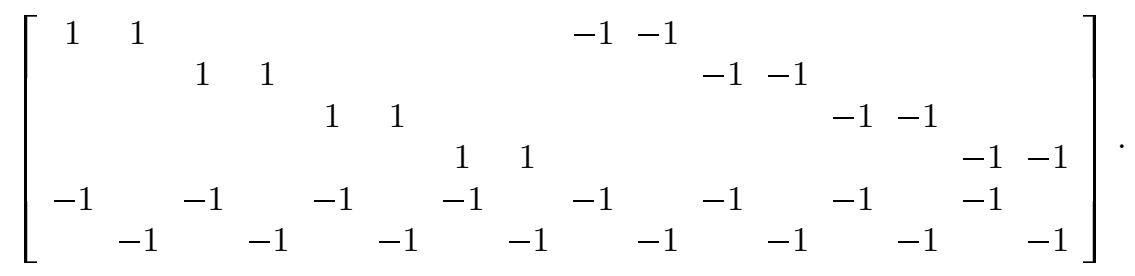

The representation spaces which are associated to the images of $\frac{1}{2}( \pm 1, \ldots, \pm 1)$ are listed in table 2. They are divided into two parts such that the first 18 summands give the decomposition of the positive spinor representation, and the second 12 summands yield the decomposition of the negative spinor representation.

TABle 2. Representations of $\mathfrak{s o}(8) \oplus \mathfrak{s o}(4)$

\begin{tabular}{c|c|c} 
Representation of & Irreducible Dec. w.r.t. & \\
$\mathfrak{s o}(8) \oplus \mathfrak{s o}(4)$ & $\mathfrak{s o}(8) \oplus \mathfrak{s u}(2) \oplus \mathfrak{s u}(2)$ & Dimension \\
\hline \hline$\left(0000 \mid 44^{ \pm}\right)$ & $(0000|8| 0) \oplus(0000|0| 8)$ & $2 \cdot(1 \cdot 9 \cdot 1)=2 \cdot 9$ \\
$\left(2000 \mid 33^{ \pm}\right)$ & $(2000|6| 0) \oplus(2000|0| 6)$ & $2 \cdot(35 \cdot 7 \cdot 1)=2 \cdot 245$ \\
$\left(1100 \mid 42^{ \pm}\right)$ & $(1100|6| 2) \oplus(1100|2| 6)$ & $2 \cdot(28 \cdot 7 \cdot 3)=2 \cdot 588$ \\
$\left(2200 \mid 22^{ \pm}\right)$ & $(2200|4| 0) \oplus(2200|0| 4)$ & $2 \cdot(300 \cdot 5 \cdot 1)=2 \cdot 1500$ \\
$\left(2110 \mid 31^{ \pm}\right)$ & $(2110|4| 2) \oplus(2110|2| 4)$ & $2 \cdot(350 \cdot 5 \cdot 3)=2 \cdot 5250$ \\
$\left(1111^{ \pm} \mid 40\right)$ & $\left(1111^{+}|4| 4\right) \oplus\left(1111^{-}|4| 4\right)$ & $2 \cdot(35 \cdot 5 \cdot 5)=2 \cdot 875$ \\
$\left(2220 \mid 11^{ \pm}\right)$ & $(2220|2| 0) \oplus(2220|0| 2)$ & $2 \cdot(840 \cdot 3 \cdot 1)=2 \cdot 2520$ \\
$\left(2211^{ \pm} \mid 20\right)$ & $\left(2211^{+}|2| 2\right) \oplus\left(2211^{-}|2| 2\right)$ & $2 \cdot(567 \cdot 3 \cdot 3)=2 \cdot 5103$ \\
$\left(2222^{ \pm} \mid 00\right)$ & $\left(2222^{+}|0| 0\right) \oplus\left(2222^{-}|0| 0\right)$ & $2 \cdot(294 \cdot 1 \cdot 1)=2 \cdot 294$ \\
\hline$\left(1000 \mid 43^{ \pm}\right)$ & $(1000|7| 1) \oplus(1000|1| 7)$ & $2 \cdot(8 \cdot 8 \cdot 2)=2 \cdot 128$ \\
$\left(2100 \mid 32^{ \pm}\right)$ & $(2100|5| 1) \oplus(2100|1| 5)$ & $2 \cdot(160 \cdot 6 \cdot 2)=2 \cdot 1920$ \\
$\left(1110 \mid 41^{ \pm}\right)$ & $(1110|5| 3) \oplus(1110|3| 5)$ & $2 \cdot(56 \cdot 6 \cdot 4)=2 \cdot 1344$ \\
$\left(2210 \mid 21^{ \pm}\right)$ & $(2210|3| 1) \oplus(2210|1| 3)$ & $2 \cdot(840 \cdot 4 \cdot 2)=2 \cdot 6720$ \\
$\left(2111^{ \pm} \mid 30\right)$ & $\left(2111^{+}|3| 3\right) \oplus\left(2111^{-}|3| 3\right)$ & $2 \cdot(224 \cdot 4 \cdot 4)=2 \cdot 3584$ \\
$\left(2221^{ \pm} \mid 10\right)$ & $\left(2221^{+}|1| 1\right) \oplus\left(2221^{-}|1| 1\right)$ & $2 \cdot(672 \cdot 2 \cdot 2)=2 \cdot 2688$ \\
\hline & &
\end{tabular}

$\underline{k=\ell=3:}$ : In this example the matrix which obeys $\left(6 K_{i}^{*}, 6 L_{j}^{*}\right)=A\left(\epsilon_{i j}^{e}, \epsilon_{i j}^{f}\right)$ has size $6 \times 18$. As before, we list the irreducible representation spaces which are associated to the images of $\frac{1}{2}( \pm 1, \ldots, \pm 1)$. The result for $S^{+}$can be found in table 3 . The representations for $S^{-}$can be obtained by interchanging the two factors. 
TABLE 3. Representations of $\mathfrak{s o}(6) \oplus \mathfrak{s o}(6)$

\begin{tabular}{c|c}
$\begin{array}{c}\text { Irreducible Rep. of } \\
\mathfrak{s}(6) \oplus \mathfrak{s o}(6)\end{array}$ & Dimension \\
\hline \hline$\left(000 \mid 333^{ \pm}\right)$ & $2 \cdot(1 \cdot 84)=2 \cdot 84$ \\
$\left(110 \mid 331^{ \pm}\right)$ & $2 \cdot(15 \cdot 270)=2 \cdot 4050$ \\
$\left(200 \mid 322^{ \pm}\right)$ & $2 \cdot(20 \cdot 140)=2 \cdot 2800$ \\
$\left(211^{ \pm} \mid 320\right)$ & $2 \cdot(45 \cdot 300)=2 \cdot 13500$ \\
$\left(220 \mid 311^{ \pm}\right)$ & $2 \cdot(84 \cdot 126)=2 \cdot 10584$ \\
$\left(222^{ \pm} \mid 300\right)$ & $2 \cdot(35 \cdot 50)=2 \cdot 1750$ \\
$\left(332^{ \pm} \mid 100\right)$ & $2 \cdot(6 \cdot 189)=2 \cdot 1134$ \\
$\left(330 \mid 111^{ \pm}\right)$ & $2 \cdot(10 \cdot 300)=2 \cdot 3000$ \\
$\left(321^{ \pm} \mid 210\right)$ & $2 \cdot(64 \cdot 256)=2 \cdot 16384$ \\
$\left(310 \mid 221^{ \pm}\right)$ & $2 \cdot(70 \cdot 175)=2 \cdot 12250$ \\
& $=\frac{1}{2} \cdot 2^{18}$
\end{tabular}

Before we state the general result we introduce the following operation.

Definition 10. Let $\lambda=\left(\lambda_{1}, \ldots, \lambda_{n}\right)$ be a vector with non-negative decreasing integer entries. For $m \geqslant \max \left\{\lambda_{j}\right\}, \ell \geqslant n$ the $(\ell, m)$-conjugat $5 \lambda^{c(\ell, m)}$ is defined by the vector which represents the Young diagram obtained by the following procedure. Extend the Young diagram associated to $\lambda$ to an rectangle of size $(\ell \times m)$, erase $\lambda$, rotate the remaining part by $180^{\circ}$, and reflect at the main diagonal.

$$
\left(\lambda^{c(\ell, m)}\right)_{j}=\ell-\#\left\{i \mid \lambda_{i} \geqslant m-j+1\right\} \quad \text { for } 1 \leqslant j \leqslant m .
$$

For example, $(5,3,2,0)^{c(4,6)}=(4,3,3,2,1,1)$ :
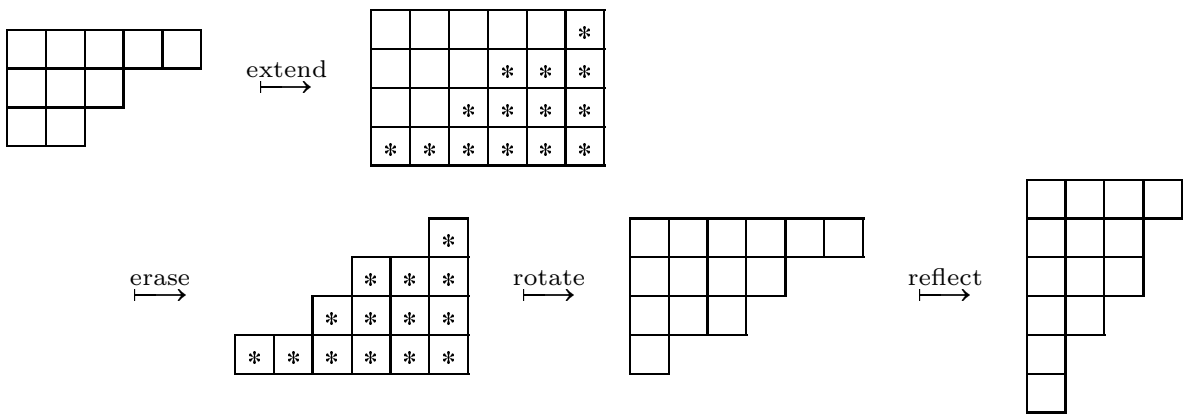

Remark 11. - We denote by $\lambda^{\prime}$ the transpose of the diagram $\lambda$, i.e., the reflection of $\lambda$ at the main diagonal. Then the $(\ell, m)$-conjugate and the transpose

${ }^{5}$ We may always assume $n=\ell$ by extending $\lambda$ by zeros. 
are connected by

$$
\lambda_{j}^{c(\ell, m)}=\ell-\lambda_{m-j+1}^{\prime}, \quad 1 \leqslant j \leqslant m .
$$

- Furthermore we have

$$
\left(\lambda^{\prime}\right)^{\prime}=\left(\lambda^{c(\ell, m)}\right)^{c(m, \ell)}=\lambda
$$

and therefore

$$
\left(\lambda^{c(\ell, m)}\right)_{j}^{\prime}=m-\lambda_{\ell-j+1}, \quad 1 \leqslant j \leqslant \ell .
$$

- For $\lambda=\left(\lambda_{1}, \ldots, \lambda_{\ell}\right)$ we have

$$
\lambda^{c(\ell, m)}=\left(\ell^{m-\lambda_{1}},(\ell-1)^{\lambda_{1}-\lambda_{2}}, \ldots, 1^{\lambda_{\ell-1}-\lambda_{\ell}}, 0^{\lambda_{\ell}}\right) .
$$

Conjecture 12. Let $G_{2 k, 2 \ell}=S O(2(k+\ell)) /(S O(2 k) \times S O(2 \ell))$ be the spin Grassmannian of even dimension $4 k \ell$ and let $S^{+}$and $S^{-}$the positive and negative spinor bundles respectively. Suppose $k \geqslant \ell$. The spinor bundle $S^{+} \oplus S^{-}$decomposes into a sum of $2 \cdot\left(\begin{array}{c}k+\ell \\ \ell\end{array}\right)$ subbundles associated to irreducible representations of $\mathfrak{s o}(2 k) \oplus \mathfrak{s o}(2 \ell)$. The weights of these representations are given by

$$
\left(\lambda_{1}, \ldots, \lambda_{k} \mid \mu_{1}, \ldots, \mu_{\ell}\right),
$$

with $\ell \geqslant \lambda_{1} \geqslant \cdots \geqslant \lambda_{k-1} \geqslant\left|\lambda_{k}\right| \geqslant 0, k \geqslant \mu_{1} \geqslant \cdots \geqslant \mu_{\ell-1} \geqslant\left|\mu_{\ell}\right| \geqslant 0$ and

$$
\left(\lambda_{1}, \ldots,\left|\lambda_{k}\right|\right)=\left(\mu_{1}, \ldots,\left|\mu_{\ell}\right|\right)^{c(\ell, k)},
$$

or equivalently

$$
\left(\mu_{1}, \ldots,\left|\mu_{\ell}\right|\right)=\left(\lambda_{1}, \ldots,\left|\lambda_{k}\right|\right)^{c(k, \ell)} .
$$

In particular $\sum_{i=1}^{k} \lambda_{i}+\sum_{j=1}^{\ell} \mu_{j}=k \ell$.

Theorem 13. Conjecture 12 is true for $\ell=2$.

Remark 14. - For $\lambda_{k} \neq 0$ (in this case $\mu_{\ell}=0$ ) the representation with $-\lambda_{k}$ is present within the decomposition, too. This symmetry is due to self duality with respect to $\mathfrak{s o}(2 k)$. This argument is symmetric with respect to the two factors $\mathfrak{s o}(2 k)$ and $\mathfrak{s o}(2 \ell)$ so that $S^{ \pm}$splits itself into two subbundles $S^{ \pm}=S^{ \pm+} \oplus S^{ \pm-}$.

- If the product $k \ell$ is even, representations with weights such that both sums $\sum_{i=1}^{k} \lambda_{i}$ and $\sum_{j=1}^{\ell} \mu_{j}$ are even (or odd) belong to $S^{+}$(or $S^{-}$respectively). In particular, $\left(0^{k} \mid k^{\ell}\right)$ and $\left(\ell^{k} \mid 0^{\ell}\right)$ belong to $S^{+}$.

- If the product $k \ell$ is odd, representations with $\sum_{i=1}^{k} \lambda_{i}$ even (or odd) and $\sum_{j=1}^{\ell} \mu_{j}$ odd (or even) belong to $S^{+}$(or $S^{-}$respectively). In particular $\left(0^{k} \mid k^{\ell}\right)$ belongs to $S^{+}$and $\left(\ell^{k} \mid 0^{\ell}\right)$ belongs to $S^{-}$.

Towards a proof. Firstly we have to show that all the mentioned weights appear as an image of $A$ on the set $\left\{\left( \pm \frac{1}{2}, \ldots, \pm \frac{1}{2}\right)\right\}$. Consider the $\mathfrak{s o}(2 \ell)$-representation $\lambda=\left(\lambda_{1}, \ldots, \lambda_{\ell}\right)$ with $\lambda_{\ell} \geqslant 0$. We associate vectors $\vec{f}_{\mu} \in \mathbb{R}^{\ell}$ and $\vec{w} \in \mathbb{R}^{2 k l}$ given by

$$
2 \vec{f}_{i}=(\overbrace{-1, \ldots,-1}^{i}, 1, \ldots, 1), \quad 0 \leqslant i \leqslant \ell
$$

and

$$
\vec{w}=(\underbrace{\vec{f}_{0}, \ldots, \vec{f}_{0}}_{k-\lambda_{1}}, \ldots, \underbrace{\vec{f}_{i}, \ldots, \vec{f}_{i}}_{\lambda_{i}-\lambda_{i+1}}, \ldots, \underbrace{\vec{f}_{\ell}, \ldots, \vec{f}_{\ell}}_{k+\lambda_{\ell}}) .
$$


to the tuple $\lambda$. Then $\vec{w}$ is a preimage of

$$
\left(\ell^{k-\lambda_{1}},(\ell-1)^{\lambda_{1}-\lambda_{2}}, \ldots, 1^{\lambda_{\ell-1}-\lambda_{\ell}}, 0^{\lambda_{\ell}} \mid \lambda_{1}, \ldots, \lambda_{\ell}\right)
$$

under the action of $A$ with rows given by (24).

The prove of the statement now is done by a dimension check: For $\lambda$ with $\lambda_{k} \geqslant 0$ we write $\tilde{\lambda}=\lambda^{c(k, \ell)}$ such that $\tilde{\tilde{\lambda}}=\lambda$. The dimension of the $\mathfrak{s o}(2 k)$-irrep with highest weight $\lambda=\left(\lambda_{1}, \ldots, \lambda_{k}\right)$ and the corresponding dimension of the $\mathfrak{s o}(2 \ell)$-irrep with highest weight $\tilde{\lambda}=\left(\tilde{\lambda}_{1}, \ldots, \tilde{\lambda}_{\ell}\right)$ are given by

$$
\begin{aligned}
\operatorname{dim} V_{\lambda}^{\mathfrak{s o}(2 k)} & =\prod_{1 \leqslant i<j \leqslant k} \frac{\left(\lambda_{i}+k-i\right)^{2}-\left(\lambda_{j}+k-j\right)^{2}}{(k-i)^{2}-(k-j)^{2}} \\
& =\prod_{1 \leqslant i<j \leqslant k} \frac{\left(\lambda_{i}+\lambda_{j}+2 k-i-j\right)\left(\lambda_{i}-\lambda_{j}-i+j\right)}{(2 k-i-j)(j-i)}
\end{aligned}
$$

and $(\lambda, k)$ substituted by $(\tilde{\lambda}, \ell)$. The dimension may also be expressed using the following determinant

$$
d\left(\lambda_{1}, \ldots, \lambda_{k}\right)=\operatorname{det}\left[\left(\begin{array}{c}
2 k+\lambda_{i}-i+j-1 \\
2 k-1
\end{array}\right)-\left(\begin{array}{c}
2 k+\lambda_{i}-i-j-1 \\
2 k-1
\end{array}\right)\right]_{1 \leqslant i, j \leqslant k},
$$

see [8. To prove the main statement we have to show

$$
\sum_{\lambda \subset\left(\ell^{k}\right)} \operatorname{dim} V_{\lambda}^{\mathfrak{s o}(2 k)} \cdot \operatorname{dim} V_{\tilde{\lambda}}^{\mathfrak{s o}(2 \ell)}=2^{2 k \ell-1},
$$

where we have to take one half of the dimension of the spinor module because the construction yields that either $\lambda$ or $\tilde{\lambda}$ has vanishing last component.

3.2. The case $\ell \leqslant 2$. In this section we prove theorem 13 . We recall that conjecture 12 for $\ell=1$ is shown in 27] such that we consider the case $G_{2 k, 4}$, which is one of the compact quaternionic Kähler symmetric spaces classified in [30.

We label the $\mathfrak{s o}(4)$-weights by two numbers $k \geqslant p \geqslant q \geqslant 0$ such that the spaces in the decomposition (29) have the weights

$$
\left(2^{k-p} 1^{p-q} 0^{q} \mid p q\right)^{ \pm}
$$

The dimension of the $\mathfrak{s o}(4)$-representation $(p, q)$ is

$$
\operatorname{dim} V_{(p q \pm)}^{\mathfrak{s o}(4)}=(p+q+1)(p-q+1) .
$$

We use the classical result of [18] on the characters of the classical groups to evaluate the dimensions of the representation spaces of $\mathfrak{s o}(2 k)$ which we need here.

Proposition 15. Let $\lambda$ be the highest weight of an $\mathfrak{s o}(2 k)$ representation with $\lambda_{k} \geqslant 0$, then the dimension of the representation is connected to the dimension of representations with respect to $\mathfrak{g l}(2 k)$ via the Littlewood-Richardson-coefficients LR by

$$
\operatorname{dim} V_{\lambda}^{\mathfrak{s o}(2 k)}=\sum_{\mu} \sum_{\alpha_{1}>\cdots>\alpha_{s}>0}(-1)^{\sum \alpha_{j}} L R_{\sigma(\alpha), \mu}^{\lambda} \operatorname{dim} V_{\mu}^{\mathfrak{g l}(2 k)} .
$$

where the sum also contains $\alpha=(0)$. 
Here $\sigma(\alpha)$ is the weight corresponding to the diagram which $i$ th row has $\alpha_{i}+i$ boxes and its $i$ th column has $\alpha_{i}+i-1$ boxes. The weights $\mu$ for which $L R_{\sigma(\alpha), \mu}^{\lambda} \neq 0$ correspond to traces of $\lambda$ associated to the symmetry of $\sigma(\alpha)$.

In the our case $-\lambda_{i} \leqslant 2$ - only those coefficients are nonzero for which the diagram associated to $\sigma(\alpha)$ fits into the diagram associated to $\lambda$. The possible candidates are $\alpha=(0)$ with $\sigma(\alpha)=(0, \ldots, 0)$ and $\alpha=(1)$ with $\sigma(\alpha)=(2,0, \ldots, 0)$. The weights $\mu$ such that $L R_{\sigma(1), \mu}^{\lambda} \neq 0$ are associated to the trace of $\lambda$ and if non vanishing the value of $L R$ is 1 . For $\lambda=\left(2^{a} 1^{b} 0^{c}\right)$ the trace is given by $\left(2^{a-1} 1^{b} 0^{c+1}\right)$.

If we define 6

$$
\begin{aligned}
f(k, p, q) & :=\frac{\prod_{\alpha=1}^{k-q}(2 k+1-\alpha) \prod_{\alpha=1}^{k-p}(2 k+2-\alpha)}{\prod_{\alpha=1}^{k-p}(k-q+2-\alpha) \prod_{\alpha=1}^{p-q} \alpha \prod_{\alpha=1}^{k-p} \alpha} \\
& =\frac{p-q+1}{2 k+1}\left(\begin{array}{c}
2 k+1 \\
k-q+1
\end{array}\right)\left(\begin{array}{c}
2 k+1 \\
k-p
\end{array}\right),
\end{aligned}
$$

and $f(k, k+1, q)=0$ we have for all $k \geqslant p \geqslant q \geqslant 0$,

$$
\begin{aligned}
& f(k, p, q)-f(k, p+1, q+1) \\
= & \frac{(2 k) !(2 k+2) !(p+q+1)(p-q+1)}{(k+q+1) !(k+p+2) !(k-q+1) !(k-p) !} \\
= & \frac{(p+q+1)(p-q+1)}{(2 k+1)(2 k+2)}\left(\begin{array}{c}
2 k+2 \\
k-p
\end{array}\right)\left(\begin{array}{c}
2 k+2 \\
k-q+1
\end{array}\right),
\end{aligned}
$$

so that the dimension of the $\mathfrak{s o}(2 k)$-representation $\left(2^{k-p} 1^{p-q} 0^{q}\right)^{ \pm}$is given by

$$
\operatorname{dim}\left(2^{k-p} 1^{p-q} 0^{q}\right)=f(k, p, q)-f(k, p+1, q+1) \quad \text { if } k \geqslant p \geqslant q>0,
$$

and

$$
\operatorname{dim}\left(2^{k-p} 1^{p}\right)^{ \pm}=\frac{1}{2}(f(k, p, 0)-f(k, p+1,1)) \quad \text { if } k \geqslant p \geqslant q=0 .
$$

The proof of conjecture 12 for $\ell=2$ is done by showing the identity

$$
\begin{aligned}
& \quad \sum_{0 \leqslant p \leqslant k} \frac{(p+1)^{4}}{2}\left(\begin{array}{c}
2 k+2 \\
k-p
\end{array}\right)\left(\begin{array}{c}
2 k+2 \\
k+1
\end{array}\right) \\
& \quad+\sum_{1 \leqslant q \leqslant p \leqslant k}(p+q+1)^{2}(p-q+1)^{2}\left(\begin{array}{c}
2 k+2 \\
k-p
\end{array}\right)\left(\begin{array}{c}
2 k+2 \\
k-q+1
\end{array}\right) \\
& =(2 k+2)(2 k+1) 2^{4 k-1} .
\end{aligned}
$$

The left hand side of this can be written as

$$
\begin{aligned}
& \sum_{1 \leqslant p \leqslant k+1} \frac{p^{4}}{2}\left(\begin{array}{c}
2 k+2 \\
k+1-p
\end{array}\right)\left(\begin{array}{c}
2 k+2 \\
k+1
\end{array}\right) \\
& +\sum_{1 \leqslant q<p \leqslant k+1}(p-q)^{2}(p+q)^{2}\left(\begin{array}{c}
2 k+2 \\
k+1-p
\end{array}\right)\left(\begin{array}{c}
2 k+2 \\
k+1-q
\end{array}\right) .
\end{aligned}
$$

\footnotetext{
${ }^{6}$ This is the dimension of the $\mathfrak{g l}(2 k)$ representation space with highest weight $\left(2^{k-p} 1^{p-q} 0^{q}\right)$.
} 
The terms of the second sum are symmetric with respect to $p$ and $q$ and vanish for $p=q$ so that we may sum over the whole square instead of one triangle. So we may further write

$$
\begin{aligned}
=\frac{1}{2} & \sum_{1 \leqslant p \leqslant k+1} p^{4}\left(\begin{array}{c}
2 k+2 \\
k+1-p
\end{array}\right)\left(\begin{array}{c}
2 k+2 \\
k+1
\end{array}\right) \\
& +\frac{1}{2} \sum_{1 \leqslant q, p \leqslant k+1}(p-q)^{2}(p+q)^{2}\left(\begin{array}{c}
2 k+2 \\
k+1-p
\end{array}\right)\left(\begin{array}{c}
2 k+2 \\
k+1-q
\end{array}\right) .
\end{aligned}
$$

Therefore the following lemma - which we will prove in appendix $\mathrm{A}$ - yields the subsequent theorem 13 .

\section{Lemma 16.}

$$
\begin{aligned}
& \sum_{i=0}^{n} \sum_{j=0}^{n}\left(i^{2}-j^{2}\right)^{2}\left(\begin{array}{c}
2 n \\
n-i
\end{array}\right)\left(\begin{array}{c}
2 n \\
n-j
\end{array}\right)-\left(\begin{array}{c}
2 n \\
n
\end{array}\right) \sum_{j=0}^{n} j^{4}\left(\begin{array}{c}
2 n \\
n-j
\end{array}\right) \\
= & 2^{4 n-3} n(2 n-1)
\end{aligned}
$$

This proves theorem 13 .

3.3. The odd dimensional case. We consider the odd dimensional spin Grassmannian $G_{2 k+1,2 \ell+1}=S O(2 k+2 \ell+2) / S O(2 k+1) \times S O(2 \ell+1)$. The construction is almost the same as in the even dimensional case so that we will be more brief here. For the construction we take over the notation from section 3.1 and add two more one dimensional spaces $V_{k+1}:=\operatorname{span}\left\{v_{k+1}\right\}, W_{\ell+1}:=\operatorname{span}\left\{w_{\ell+1}\right\}$. We define in addition

$$
\begin{aligned}
& E_{i, \ell+1}:=V_{i} \otimes W_{\ell+1}=\operatorname{span}\left\{e_{+}^{i, \ell+1}, e_{-}^{i, \ell+1}\right\} \quad \text { with } e_{+/-}^{i, \ell+1}:=v_{1 / 2}^{i} \otimes w_{\ell+1}, \\
& F_{k+1, i}:=V_{k+1} \otimes W_{i}=\operatorname{span}\left\{f_{+}^{k+1, i}, f_{-}^{k+1, i}\right\} \quad \text { with } f_{+/-}^{k+1, i}:=v_{k+1} \otimes w_{2 / 1}^{i} \text {, } \\
& G:=\operatorname{span}\left\{v_{k+1} \otimes w_{\ell+1}\right\} \text {. }
\end{aligned}
$$

Then $\oplus E_{i j} \oplus \oplus F_{i j} \oplus G$ yields the corresponding decomposition of $\mathbb{R}^{(2 k+1)(2 \ell+1)}$ such that the Cartan basis of $s o(4 k \ell+2 \ell+2 k+1)$ is given by $\left.\left\{N_{i j}^{e}\right\}, N_{i^{\prime} j^{\prime}}^{f}\right\}$ with

$$
\begin{aligned}
N_{i j}^{e}\left(e_{ \pm}^{i_{ \pm}^{\prime \prime} j^{\prime \prime}}\right) & = \pm \delta_{i}^{i^{\prime \prime}} \delta_{j}^{j^{\prime \prime}} e_{\bar{\mp}}^{i j} & & \text { for } 1 \leqslant i \leqslant k, 1 \leqslant j \leqslant \ell+1, \\
N_{i^{\prime} j^{\prime}}^{f}\left(f_{ \pm}^{i^{\prime \prime} j^{\prime \prime}}\right) & = \pm \delta_{i^{\prime}}^{i^{\prime \prime}} \delta_{j^{\prime}}^{j^{\prime \prime}} f_{\bar{\mp}}^{i^{\prime} j^{\prime}} & & \text { for } 1 \leqslant i^{\prime} \leqslant k+1,1 \leqslant j^{\prime} \leqslant \ell .
\end{aligned}
$$

Then we get

$$
\begin{aligned}
& K_{i}=\sum_{j=1}^{\ell}\left(N_{i j}^{e}-N_{i j}^{f}\right)+N_{i, \ell+1}^{e} \\
& L_{j}=-\sum_{i=1}^{\ell}\left(N_{i j}^{e}+N_{i j}^{f}\right)-N_{k+1, j}^{f}
\end{aligned}
$$


The corresponding matrix $A$ which is of size $(k+\ell) \times(2 k \ell+k+\ell)$ has rows

$$
\begin{aligned}
& A_{i}=(\overbrace{0, \ldots, 0}^{(i-1)(\ell+1)}, \overbrace{1 \ldots, 1}^{\ell+1}, \overbrace{0, \ldots, 0}^{(k-i)(\ell+1)}, \overbrace{0, \ldots, 0}^{(i-1) \ell}, \overbrace{-1 \ldots,-1}^{\ell}, \overbrace{0, \ldots, 0}^{(k-i) \ell}, \overbrace{0, \ldots, 0}^{\ell}),
\end{aligned}
$$

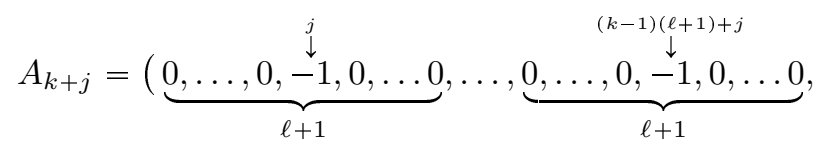

$$
\begin{aligned}
& \underbrace{0, \ldots, 0,-1,0, \ldots, 0}_{\ell}, \ldots, \underbrace{0, \ldots, 0,-1,0, \ldots, 0}_{\ell}),
\end{aligned}
$$

for $1 \leqslant i \leqslant k$ and $1 \leqslant j \leqslant \ell$. For example,

$$
\begin{gathered}
A^{k=\ell=1}=\left(\begin{array}{cccc}
1 & 1 & -1 & 0 \\
-1 & 0 & -1 & -1
\end{array}\right), \quad A^{k=2, \ell=1}=\left(\begin{array}{ccccccccc}
1 & 1 & 0 & 0 & -1 & 0 & 0 \\
0 & 0 & 1 & 1 & 0 & -1 & 0 \\
-1 & 0 & -1 & 0 & -1 & -1 & -1
\end{array}\right), \\
A^{k=\ell=2}=\left(\begin{array}{cccccccccccc}
1 & 1 & 1 & 0 & 0 & 0 & -1 & -1 & 0 & 0 & 0 & 0 \\
0 & 0 & 0 & 1 & 1 & 1 & 0 & 0 & -1 & -1 & 0 & 0 \\
-1 & 0 & 0 & -1 & 0 & 0 & -1 & 0 & -1 & 0 & -1 & 0 \\
0 & -1 & 0 & 0 & -1 & 0 & 0 & -1 & 0 & -1 & 0 & -1
\end{array}\right) .
\end{gathered}
$$

In contrast to the case of even dimensional spin Grassmannians we have the following facts on the images.

Remark 17 . The image of the set $\left\{\frac{1}{2}( \pm 1, \ldots \pm 1)^{T}\right\}$ by the map $A$ consist of weights which entries are contained in $\mathbb{Z}+\frac{1}{2}$.

Conjecture 18. The spinor bundle of the odd dimensional spin Grassmannian decomposes into a sum of $\left(\begin{array}{c}k+\ell \\ \ell\end{array}\right)$ summands which are irreducible with respect to $\mathfrak{s o}(2 k+1) \oplus \mathfrak{s o}(2 \ell+1)$ and which are associated to the weights

$$
\left(\lambda_{1}+\frac{1}{2}, \ldots, \lambda_{k}+\frac{1}{2} \mid \mu_{1}+\frac{1}{2}, \ldots \mu_{\ell}+\frac{1}{2}\right),
$$

with $0 \leqslant \lambda_{1} \leqslant \ldots \leqslant \lambda_{k} \leqslant \ell, 0 \leqslant \mu_{1} \leqslant \ldots \leqslant \mu_{\ell} \leqslant k$, and

$$
\left(\lambda_{1}, \ldots, \lambda_{k}\right)^{c(k, \ell)}=\left(\mu_{1}, \ldots, \mu_{\ell}\right) \text {, or }\left(\mu_{1}, \ldots, \mu_{\ell}\right)^{c(\ell, k)}=\left(\lambda_{1}, \ldots, \lambda_{k}\right) .
$$

Theorem 19. Conjecture 18 is true for $\ell \leqslant 2$.

That all the mentioned weights appear as image can be seen as in the even dimensional case by constructing an explicit preimage. The dimension argument uses the following observation (see, e.g., [5]).

Proposition 20. Let $\vec{e}:=(1, \ldots, 1)$. Then the dimension of the $\mathfrak{s o}(2 k+1)$ representation $V_{\lambda+\frac{1}{2} \vec{e}}^{\mathfrak{s o}(2 k+1)}$ associated to the weight $\lambda+\frac{1}{2} \vec{e}$ with $\lambda$ integer valued is given by

$$
\operatorname{dim} V_{\lambda+\frac{1}{2} \vec{e}}^{\mathfrak{s o}(2 k+1)}=2^{k} \operatorname{dim} V_{\lambda}^{\mathfrak{s p}(2 k)},
$$

where $V_{\lambda}^{\mathfrak{s p}(2 k)}$ is the $\mathfrak{s p}(2 k)$-representation associated to the weight $\lambda$.

So we end up with an equation to verify which is similar to (31)

$$
\sum_{\lambda \in\left(\ell^{k}\right)} \operatorname{dim} V_{\lambda}^{\mathfrak{s p}(2 k)} \cdot \operatorname{dim} V_{\lambda^{c}}^{\mathfrak{s p}(2 \ell)}=2^{2 k \ell}
$$


3.4. The case $\ell \leqslant 2$. In the odd dimensional case beside our strong belief that the decomposition is true in general we have the following partial result.

Proof. $\ell=1$ : The representations which play a role are $(\lambda)$ with $1 \leqslant \lambda \leqslant k$ and $\lambda^{c}=\left(1^{k-\lambda}\right)$ with dimensions

$$
\operatorname{dim} V_{\lambda}^{\mathfrak{s p}(2)}=\lambda+1, \quad \operatorname{dim} V_{\left(1^{m}\right)}^{\mathfrak{s p}(2 k)}=\frac{k+1-m}{k+1}\left(\begin{array}{c}
2 k+2 \\
m
\end{array}\right) .
$$

This yields

$$
\begin{aligned}
\sum_{\lambda=0}^{k} \operatorname{dim} V_{\left(1^{k-\lambda}\right)+\frac{1}{2} \vec{e}}^{\mathfrak{s o}(2 k+1)} \operatorname{dim} V_{(\lambda)+\frac{1}{2} \vec{e}}^{\mathfrak{s o}(3)} & =2^{k+1} \sum_{\lambda=0}^{k} \operatorname{dim} V_{\left(1^{k-\lambda}\right)}^{\mathfrak{s p}(2 k)} \operatorname{dim} V_{(\lambda)}^{\mathfrak{s p}(2)} \\
& =2^{k+1} \sum_{\lambda=0}^{k} \frac{(\lambda+1)^{2}}{k+1}\left(\begin{array}{c}
2 k+2 \\
k-\lambda
\end{array}\right) \\
& =2^{k+1} \frac{1}{k+1} B(k+1,2) \\
& =2^{3 k+1}
\end{aligned}
$$

$\underline{\ell=2:}$ A formula similar to (33) holds in the symplectic case

$$
\operatorname{dim} V_{\lambda}^{\mathfrak{s p}(2 k)}=\sum_{\mu} \sum_{\alpha_{1}>\cdots>\alpha_{s}>0}(-1)^{\sum \alpha_{j}} L R_{\sigma(\alpha), \mu^{\prime}}^{\lambda^{\prime}} \operatorname{dim} V_{\mu}^{\mathfrak{g l}(2 k)} .
$$

We list the relevant partitions $\mu$ which enter into the dimension formula in the symplectic case when we start with $\left(2^{k-p}, 1^{p-q}, 0^{q}\right)$.

\begin{tabular}{l|l|l|l}
$\alpha$ & $\sigma(\alpha)$ & $\mu$ & condition \\
\hline \hline$(1)$ & $\square$ & $\left(2^{k-p}, 1^{p-q-2}, 0^{q+2}\right)$ & $p-q \geqslant 2$ \\
& & $\left(2^{k-(p+1)}, 1^{p-q}, 0^{q+1}\right)$ & $p-q \geqslant 1$ \\
& & $\left(2^{k-(p+2)}, 1^{p-q+2}, 0^{q}\right)$ & none \\
\hline$(2)$ & $\square$ & $\left(2^{k-(p+1)}, 1^{p-q-2}, 0^{q+3}\right)$ & $p-q \geqslant 2$ \\
& & $\left(2^{k-(p+2)}, 1^{p-q}, 0^{q+2}\right)$ & $p-q \geqslant 1$ \\
& & $\left(2^{k-(p+3)}, 1^{p-q+2}, 0^{q+1}\right)$ & none \\
\hline$(2,1)$ & $\square$ & $\left(2^{k-(p+3)}, 1^{p-q}, 0^{q+3}\right)$ & none
\end{tabular}

We recall the dimension of the $\mathfrak{g l}(2 k)$-representation $\left(2^{k-p}, 1^{p-q}, 0^{q}\right)$

$$
f(k, p, q)=\frac{p-q+1}{2 k+1}\left(\begin{array}{c}
2 k+1 \\
k-q+1
\end{array}\right)\left(\begin{array}{c}
2 k+1 \\
k-p
\end{array}\right),
$$

see (34). Therefore the dimension of the $\mathfrak{s p}(2 k)$-representation associated to the weight $\left(2^{k-p}, 1^{p-q}, 0^{q}\right)$ is

$$
\begin{aligned}
\operatorname{dim} V_{\left(2^{k-p}, 1^{p-q}, 0^{q}\right)}^{\mathfrak{s p}(2 k)}= & f(k, p, q)-f(k, p+3, q+3) \\
& +\sum_{b=\max \{3-(p-q), 1\}}^{3} f(k, p+b, q+4-b) \\
& -\sum_{b=\max \{2-(p-q), 0\}}^{2} f(k, p+b, q+2-b) .
\end{aligned}
$$

\footnotetext{
${ }^{7}$ We recall that $\lambda^{\prime}$ is the diagram transpose to $\lambda$.
} 
Furthermore we have

$$
\begin{aligned}
\operatorname{dim} V_{(p, q)}^{\mathfrak{s p}(4)} & =\operatorname{dim} V_{(p, q)}^{\mathfrak{g l}(4)}-\operatorname{dim} V_{(p-1, q-1)}^{\mathfrak{g l}(4)} \\
& =\frac{1}{6}(p-q+1)(p+q+3)(p+2)(q+1) .
\end{aligned}
$$

So we have to show

$$
\sum_{0 \leqslant q \leqslant p \leqslant k} \operatorname{dim} V_{\left(2^{k-p}, 1^{p-q}, 0^{q}\right)}^{\mathfrak{s p}(2 k)} \operatorname{dim} V_{(p, q)}^{\mathfrak{s p}(4)}=2^{4 k} .
$$

This is done by expanding the left hand side which - after a lengthy calculation performed in appendix $B$ - turns it into a sum of products of at most two of the terms $B(k+1, m)$ for $m \leqslant 5$, see (59) in appendix $\mathrm{A}$.

\section{On the spectrum And the EIGenspaces of the DiraC operator}

4.1. General symmetric spaces. The construction described in section 2.1 is used to calculate the spectrum of the eigenvalues of Dirac operators. This has been performed in 25] in general and we give a short review. The $L^{2}$-sections of the spinor bundle on $G / K$ are identified with the $K$-equivariant maps from $G$ to the spinor module $\Delta$. Due to Frobenius reciprocity this can be further identified with $\operatorname{Hom}_{K}(\mathbb{C} G, \Delta)=\bigoplus_{\lambda \in \operatorname{Irrep}(G)} V_{\lambda} \otimes \operatorname{Hom}_{K}\left(V_{\lambda}, \Delta\right)$ (see [8]). The square of the Dirac operator acts via $\not \not^{2}=C_{\lambda}+\frac{s}{8}$ where the Casimir $C_{\lambda}=c_{\lambda} \mathbb{1}$ acts proportionally to the identity due to the irreducibility of the representations. The factor is $c_{\lambda}=$ $b\left(\lambda+2 \alpha_{\mathfrak{g}}, \lambda\right)=\left\|\lambda+\alpha_{\mathfrak{g}}\right\|^{2}-\left\|\alpha_{\mathfrak{g}}\right\|^{2}$, where $\alpha_{\mathfrak{g}}$ is the weight (4) and $b$ is the metric induced by the Killing form of $\mathfrak{g}$. Using this the spectrum of the square of the Dirac operator is given by

$$
\operatorname{spec}\left(\not \not^{2}\right)=\left\{c_{\lambda}+\frac{s}{8} \mid \lambda \in \mathcal{V}(G / K)\right\},
$$

where the condition on the weight of the used $G$-representations is

$$
\mathcal{V}(G / K):=\left\{\begin{array}{l|l}
\lambda & \begin{array}{l}
\lambda \text { is highest weight of a } G \text {-irrep. s.t. one sum- } \\
\text { mand in its dec. w.r.t. } K \text { is contained in the } \\
\text { spinor dec. (33). }
\end{array}
\end{array}\right\} .
$$

In general the eigenvalue is degenerated in the sense that there exist $\left\{\lambda_{j}\right\}_{j=1, \ldots, N}$ such that $c_{\lambda_{1}}=\cdots=c_{\lambda_{N}}$.

The described construction makes use of branching rules for Lie algebras which usually are very hard to find. Nevertheless the theoretic basics provided so far can be used to calculate explicit examples as well as to formulate further general statements on the eigenvalue of the Dirac operator with the smallest absolute value (see, for example, [10, 26], 22], 24, [3] or [23]).

In the following we will make some comments on the spectrum and the eigenspaces of the Dirac operator on the Grassmann manifold.

4.2. The even dimensional Grassmannians. Given the decomposition of the spinor representation of the Grassmannian $\left.G_{2 k, 2 \ell}=S O(2 k+2 \ell)\right) /(S O(2 k) \times$ 
$S O(2 \ell))$ with respect to $\mathfrak{k}=\mathfrak{s o}(2 k) \oplus \mathfrak{s o}(2 \ell)$ as in conjecture 12. Let $\mathfrak{g}=\mathfrak{s o}(2(k+\ell))$ with $k \geqslant \ell \geqslant 2$ and consider $\alpha_{\mathfrak{k}}$ and $\alpha_{\mathfrak{g}}$ as in (4). We have

$$
\begin{aligned}
\alpha_{\mathfrak{g}} & =\sum_{i=1}^{k+\ell}(k+\ell-i) e_{i}, \\
\alpha_{\mathfrak{k}} & =\sum_{i=1}^{k}(k-i) e_{i}+\sum_{i=1}^{\ell}(\ell-i) e_{k+i} .
\end{aligned}
$$

Let $\Pi$ be the set of highest weights corresponding to the decomposition of the spinor representation (3). Furthermore let $\Psi:=\left\{\gamma \in \Phi_{\mathfrak{g}}^{+} \mid\left\langle\gamma, \alpha_{\mathfrak{k}}\right\rangle<0\right\}$

Due to [23] and 24] we are able to calculate at least the square of the smallest eigenvalue $\epsilon_{0}$ of the Dirac operator as

$$
\begin{aligned}
\epsilon_{0}^{2} & =2 \min _{\beta \in \Pi}\|\beta\|^{2}+\frac{k \ell}{2} \\
& =2\left\|\alpha_{\mathfrak{g}}-\alpha_{\mathfrak{k}}\right\|^{2}+4 \sum_{\gamma \in \Psi}\left\langle\gamma, \alpha_{\mathfrak{k}}\right\rangle+\frac{k \ell}{2} .
\end{aligned}
$$

Here $\langle\cdot, \cdot\rangle$ is the metric $b_{i j}=\frac{1}{4(k+\ell-1)} \delta_{i j}$ induced on the dual space by the Killing form (see [8]).

We observe that $\left\langle e_{i}+e_{j}, \alpha_{\mathfrak{k}}\right\rangle>0$ for all $i, j$ and $\left\langle e_{i}-e_{j}, \alpha_{\mathfrak{k}}\right\rangle<0$ only if $1 \leqslant i \leqslant k$, $k+1 \leqslant j \leqslant k+\ell$. More precisely we have

$$
\left\langle\alpha_{\mathfrak{k}}, e_{i}-e_{k+j}\right\rangle_{\text {Eucl. }}=k-i-\ell+j,
$$

such that

$$
\Psi=\left\{e_{i}-e_{k+j} \mid 1 \leqslant i \leqslant k, 1 \leqslant j \leqslant \ell, i>k-\ell+j\right\} .
$$

We have $\left\|\alpha_{\mathfrak{g}}-\alpha_{\mathfrak{k}}\right\|_{\text {Eucl. }}^{2}=\left\|\ell \sum_{i=1}^{k} e_{i}\right\|_{\text {Eucl. }}^{2}=k \ell^{2}$, so that we get

$$
\begin{aligned}
4(k+\ell-1)\left(\epsilon_{0}^{2}-\frac{k \ell}{2}\right) & =2 k \ell^{2}+4 \sum_{i=k-\ell+2}^{k} \sum_{j=1}^{i-1-(k-\ell)}(j-i+(k-\ell)) \\
& =\frac{2}{3}\left(3 k \ell^{2}-\left(\ell^{2}-1\right) \ell\right) .
\end{aligned}
$$

Example 21. Suppose $\ell=2$. Writing $k=\frac{m}{2}$ we recover the result in 24].

In particular $k=4, \ell=2$ yields $\epsilon_{0}^{2}-\frac{k \ell}{2}=\frac{2 \cdot 14}{4(k+\ell-1)}$ where $14=\|(2220 \mid 11)\|_{\text {Eucl. }}^{2}=$ $\|(2210 \mid 21)\|_{\text {Eucl. }}^{2}=\|(2221 \mid 10)\|_{\text {Eucl. }}^{2}=\|(2211 \mid 20)\|_{\text {Eucl. }}^{2}$ is the minimum of the Euclidean norms of the weights from table 2 .

With $k=\ell=2$ we get $\epsilon_{0}^{2}-\frac{k \ell}{2}=\frac{2 \cdot 6}{4(k+\ell-1)}$. Here 6 is the minimum of the Euclidean lengths of the weights corresponding to (26). There are four of them, e.g., $6=$ $\|(11 \mid 20)\|_{\text {Eucl. }}^{2}$.

Example 22. $k=\ell=3$ yields $\epsilon_{0}^{2}-\frac{k \ell}{2}=\frac{2 \cdot 19}{4(k+\ell-1)}$, where 19 is the minimum of the Euclidean lengths obtained by eight weights from table 3, e.g., $19=\|(220 \mid 311)\|_{\text {Eucl. }}^{2}$. 
Proposition 23. Let $k \geqslant \ell \geqslant 2$. The square of the smallest eigenvalue of the Dirac operator on the Grassmannian $S O(2(k+\ell)) /(S O(2 k) \times S O(2 \ell))$ is given by

$$
\epsilon_{0}^{2}=\frac{3 k \ell^{2}-\left(\ell^{2}-1\right) \ell}{6(k+\ell-1)}+\frac{k \ell}{2}
$$

The examples above (in particular, example 21) can be generalized. Therefore assume $\ell \leqslant k$. The square of the Euclidean norm of a weight which appears in conjecture 12 is

$$
\begin{aligned}
& \sum_{j=1}^{\ell} \lambda_{j}^{2}+\ell^{2}\left(k-\lambda_{1}\right)+\sum_{j=1}^{\ell-1}(\ell-j)^{2}\left(\lambda_{j}-\lambda_{j+1}\right) \\
= & k \ell^{2}+\sum_{j=1}^{\ell}\left(\left(\lambda_{j}-\ell+j\right)-\frac{1}{2}\right)^{2}-\frac{\ell}{4}-\frac{\ell\left(\ell^{2}-1\right)}{3} .
\end{aligned}
$$

This yields the following conjecture and proposition.

Conjecture 24. Suppose $\ell \leqslant k$. There are $2^{\ell}$ weights which appear in the decomposition (29) of the spinor bundle of $S O(2 k+2 \ell) / S O(2 k) \times S O(2 \ell)$ and which are associated to the smallest eigenvalue of the square of the Dirac operator. They are given by

$$
\left(\ell^{k-\lambda_{1}},(\ell-1)^{\lambda_{1}-\lambda_{2}}, \ldots, 1^{\lambda_{\ell-1}-\lambda_{\ell}}, 0^{\lambda_{\ell}} \mid \lambda_{1}, \ldots, \lambda_{\ell}\right) \quad \text { with } \lambda_{j} \in\{\ell-j+1, \ell-j\} .
$$

Proposition 25. The weights which appear in the decomposition of the spinor bundle of $S O(2 k+4) / S O(2 k) \times S O(4)$ and which are associated to the smallest eigenvalue of the square of the Dirac operator are given by

$$
\left(2^{k-1} 1 \mid 10\right), \quad\left(2^{k-1} 0 \mid 11\right), \quad\left(2^{k-2} 1^{2} \mid 20\right), \quad\left(2^{k-2} 10 \mid 21\right) .
$$

Remark 26. Both formulas (45) and (46) - of which the first uses the decomposition of the spinor bundle of the $G_{2 k, 2 \ell}$ - give the same value. This is once more a justification of the correctness of conjecture 12

We recall the following relations between $\mathfrak{s o}(2 k+2 \ell)$-representations and $\mathfrak{s o}(2 k)$ as well as $\mathfrak{s o}(2 \ell)$-representations which are given by the character formulas found in [17] and [15]

$$
\begin{aligned}
\chi_{\mu}^{\mathfrak{s o}(2 k+2 \ell)} \chi_{\nu}^{\mathfrak{s o}(2 k+2 \ell)} & =\sum L R_{\tau, \mu^{\prime}}^{\mu} L R_{\tau, \nu^{\prime}}^{\nu} L R_{\mu^{\prime}, \nu^{\prime}}^{\lambda} \chi_{\lambda}^{\mathfrak{s o}(2 k+2 \ell)}, \\
\chi_{\lambda}^{\mathfrak{s o}(2 k+2 \ell)} & =\sum L R_{2 \kappa, \lambda^{\prime}}^{\lambda} L R_{\mu, \nu}^{\lambda^{\prime}} \chi_{\mu}^{\mathfrak{s o}(2 k)} \chi_{\nu}^{\mathfrak{s o}(2 \ell)} .
\end{aligned}
$$

The first formula describes how the tensor product of two representations decompose, in particular only summands appear with $\sum \lambda_{i} \leqslant \sum \mu_{i}+\sum \nu_{i}$. In the second formula, the branching rule, only summands appear for which $\mu$ and $\nu$ are contained in $\lambda$, i.e., $\sum \lambda_{i} \geqslant \sum \mu_{i}+\sum \nu_{i}$.

The eigenspaces of the Dirac operator on the Grassmann manifold admit a decomposition with respect to the $\mathfrak{s o}(2 k+2 \ell)$-representations - see (42). The ordering $<$ of weights (see, for example, [13]) induce an ordering of the associated subspaces of the eigenspaces. From formulas (50) we get the following remark.

Remark 27. Let $\lambda$ and $\mu$ be representations of $\mathfrak{s o}(2 k+2 \ell), \mathfrak{s o}(2 k)$, respectively, which associated Young diagrams we denote by the same symbol. Furthermore $\mu$ 
shall be contained in $\left(\ell^{k}\right)$ such that $\mu^{c(k, \ell)}$ is a representation of $\mathfrak{s o}(2 \ell)$. Then $\mu \otimes \mu^{c}$ is contained in the branching of $\lambda$ if and only if there exists a $\mu^{c}$-expansion $\lambda^{\prime}$ of the diagram $\mu$ such that $\lambda$ is a $2 \kappa$-expansion of $\lambda^{\prime}$. This yields a combinatorial method to enumerate the eigenvalues of the square of the Dirac operator of $G_{2 k, 2 \ell}$. 1 . Let $N(k, \ell)$ be the number of different $\mu^{c}$-expansions of $\mu$ for $\mu \subset\left(\ell^{k}\right)$. 2. For the expansions $\left\{\lambda_{i}^{\prime}\right\}_{i=1, \ldots, N(k, \ell)}$ consider the different $2 \kappa$-expansions for all diagrams $\kappa$. 3 . From the resulting diagrams $\lambda$ calculate $c_{\lambda}$. Even in the case $G_{2 k, 4}$ this method seems to be very complex and one may suggest an analog approach as in 22 or [28] - in particular, because the ranks of the algebras are similar as in the first citation. But this fails due to the more complicated determinants which enter into the calculations so that the combinatorial ansatz is more applicable.

Example 28. For $k=\ell=2$ we have $\lambda^{\prime} \in\{\square, \square, \square\}$ such that the first $2 \kappa$ expansions which branching admits a summand from (26) are from the following list.

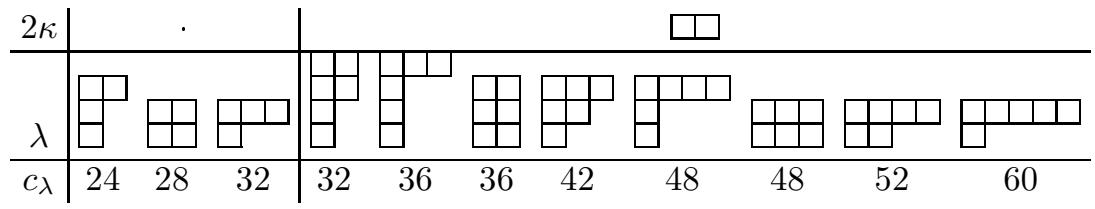

In particular, the weight with minimal $c_{\lambda}$ must obey $\lambda_{1} \leqslant 2$ and therefore the minimal eigenvalue is nondegenerated, i.e., the $\mathfrak{s o}(8)$-representation $\lambda=(2,1,1,0)$ is the unique contribution to the eigenspace associated to the minimal eigenvalue.

We turn back to the smallest eigenvalue and notice that a contribution to the eigenspace of the Dirac operator to the smallest eigenvalue must obey

$$
\begin{aligned}
\left\|\lambda+\alpha_{\mathfrak{s o}(2 k+2 \ell)}\right\|_{\text {Eucl }}^{2}-\left\|\alpha_{\mathfrak{s o}(2 k+2 \ell)}\right\|_{\text {Eucl }}^{2} & =3 k \ell^{2}+k^{2} \ell-k \ell-\frac{2}{3} \ell\left(\ell^{2}-1\right) \\
& \stackrel{\ell=2}{=} 2 k^{2}+10 k-4 .
\end{aligned}
$$

Conjecture 29. The smallest contribution to the eigenspace of the smallest eigenvalue of the square of the Dirac operator on $G_{2 k, 2 \ell}$ is given by the weight

$$
\lambda^{0}=\left(\ell^{k-\ell+1},(\ell-1)^{2},(\ell-2)^{2}, \ldots, 1^{2}, 0\right) .
$$

Proposition 30. Conjecture 29] is true for $\ell=2$.

Proof. Due to (50) the smallest contribution to the eigenspace comes from the biggest contribution in the tensor product. If we take the weights from conjecture 24 the ordering $<$ of the weights then sorts out the smallest one, i.e., the one with $\lambda_{1} \leqslant$ $\ell$. A straightforward calculation shows that $c_{\lambda^{0}}^{\text {Eucl }}=3 k \ell^{2}+k^{2} \ell-k \ell-\frac{2}{3} \ell\left(\ell^{2}-1\right)$.

Example 31. Consider the case $\ell=2$. The representations which we obtain by decomposing the tensor products $(\mu \mid \nu)$ from (49) are given in the next table where 
we also added $c_{\lambda}$.

\begin{tabular}{l|l}
$\lambda$ & $c_{\lambda}^{\text {Eucl }}$ \\
\hline$\left(4,3,2^{k-4}, 1,0^{3}\right)$ & $2 k^{2}+16 k$ \\
$\left(4,2^{k-2}, 0^{3}\right)$ & $2 k^{2}+14 k+4$ \\
$\left(4,2^{k-3}, 1^{2}, 0^{2}\right)$ & $2 k^{2}+14 k$ \\
$\left(3^{2}, 2^{k-3}, 0^{3}\right)$ & $2 k^{2}+14 k$ \\
$\left(3,2^{k-2}, 1,0^{2}\right)$ & $2 k^{2}+12 k$ \\
$\left(3,2^{k-3} 1^{3}, 0\right)$ & $2 k^{2}+12 k-6$ \\
$\left(2^{k}, 0^{2}\right)$ & $2 k^{2}+10 k$ \\
$\left(2^{k-1} 1^{2}, 0\right)$ & $2 k^{2}+10 k-4$
\end{tabular}

We see, that in this case $\lambda^{0}$ is unique, if we restrict to the weights coming from tensor products.

\section{Concluding Remarks}

Remark on the general proof of (31) and (38). In principle, all we need for the proof of conjectures 12 and 18 for fixed $\ell \geqslant 3$ is appendix $\mathrm{A}$ and the dimension formulas (33) and (39). This worked very well in the cases $\ell \leqslant 2$. In (31) and (38) the dimension of $V_{\lambda}$ for $\lambda=\left(\lambda_{1}, \ldots, \lambda_{\ell}\right)$ is a polynomial depending on the $\ell$ entries. If we use (28) to describe $\lambda^{c(\ell, k)}$ and insert the dimension formulas (33) or (39) as well as (34) into (31) or (38) we end up with sums of at most $\ell$-fold products of terms of the form $B(k+1, m)$ with $m$ increasing for $\ell$ increasing. For large $\ell$ - beside the calculation of $B(k+1, m)$ for large $m$ (which may be done by computer using the generating function (55) ) - the main difficulties arise when we try to describe uniformly the $\mu$ such that $L R_{\sigma(\alpha), \mu}^{\lambda^{c}}$ do not vanish. In particular, this problem limits the practical application of our ansatz for the proof.

Remark on the remaining case $\mathfrak{s o}(2 k) \oplus \mathfrak{s o}(2 \ell+1) \subset \mathfrak{s o}(4 k \ell+2 k)$. One thing which has been left out in section 3 is the decomposition of the spin representation of $S O(4 k \ell+2 k)$ with respect to the subgroup $S O(2 k) \times S O(2 \ell+1)$ where the embedding is such that the vector representation decomposes as $\mathbf{2} \mathbf{k} \otimes(\mathbf{2} \ell+\mathbf{1})$. We did not mention this because the symmetric space $G_{2 k, 2 \ell+1}$ is not spin. Nevertheless on the level of representation the question of branching is interesting as well. We will only state the result which is obtained by the same method as used in the two spin cases. In particular, the cases $(2 k, 3),(2 k, 5)$, and $(4,2 \ell+1)$ can be proven in the same way and the remark extends to this case, too.

The spin representation $S=S^{+} \oplus S^{-}$of $\mathfrak{s o}(4 k \ell+2 k)$ branches with respect to $\mathfrak{s o}(2 k) \oplus \mathfrak{s o}(2 \ell+1)$ into the sum of the following representations:

$$
\left(\lambda+\frac{1}{2} \vec{e}^{ \pm} \mid \lambda^{c}\right) \text { with } \lambda=\left(\lambda_{1} \ldots, \lambda_{k}\right) \subset\left(\ell^{k}\right),
$$

where $\lambda+\frac{1}{2} \vec{e}^{ \pm}=\left(\lambda_{1}+\frac{1}{2}, \ldots, \lambda_{k-1}+\frac{1}{2}, \pm\left(\lambda_{k}+\frac{1}{2}\right)\right)$ belongs to the subrepresentation $S^{ \pm}$.

Acknowledgments: I would like to thank Lorenz Schwachhöfer for useful comments and Doron Zeilberger for suggesting a method to calculate (52) for $k$ even without generating functions. 


\section{Appendix A. Useful BINOMIAL IDENTITIES}

We define sums

$$
B(n, k):=\sum_{j=0}^{n} j^{k}\left(\begin{array}{c}
2 n \\
n-j
\end{array}\right),
$$

such that lemma 16 in these terms reads as

$$
\text { (36) } 2 B(n, 4) B(n, 0)-2 B(n, 2)^{2}-\left(\begin{array}{c}
2 n \\
n
\end{array}\right) B(n, 4)=2^{4 n-3} n(2 n-1) \text {. }
$$

We provide the tools to calculate (52) for all values of $n$ and $k$ although for the proof of (36) and (40) we only need them for values less than 6 .

We consider polynomials

$$
P_{n}(x):=\sum_{i=0}^{n}\left(\begin{array}{c}
2 n \\
n-i
\end{array}\right) x^{i}
$$

which obey

$$
\begin{aligned}
P_{n}(1) & =B(n, 0), \\
P_{n}^{\prime}(1) & =B(n, 1), \\
P_{n}^{\prime \prime}(1) & =B(n, 2)-B(n, 1), \\
P_{n}^{\prime \prime \prime}(1) & =B(n, 3)-3 B(n, 2)+2 B(n, 1), \\
P_{n}^{\prime \prime \prime \prime}(1) & =B(n, 4)-6 B(n, 3)+11 B(n, 2)-6 B(n, 1), \\
P_{n}^{\prime \prime \prime \prime \prime}(1) & =B(n, 5)-10 B(n, 4)+35 B(n, 3)-50 B(n, 2)+24 B(n, 1),
\end{aligned}
$$

and

$$
\begin{aligned}
P_{n+1}(x)= & \sum_{j=0}^{n+1}\left(\begin{array}{c}
2 n+2 \\
n+1-j
\end{array}\right) x^{j} \\
= & \sum_{j=0}^{n+1}\left(\left(\begin{array}{c}
2 n \\
n-j+1
\end{array}\right)+2\left(\begin{array}{c}
2 n \\
n-j
\end{array}\right)+\left(\begin{array}{c}
2 n \\
n-j-1
\end{array}\right)\right) x^{j} \\
= & x \sum_{j=0}^{n}\left(\begin{array}{c}
2 n \\
n-j
\end{array}\right) x^{j}+2 \sum_{j=0}^{n}\left(\begin{array}{c}
2 n \\
n-j
\end{array}\right) x^{j}+\frac{1}{x} \sum_{j=0}^{n}\left(\begin{array}{c}
2 n \\
n-j
\end{array}\right) x^{j} \\
& +\left(\begin{array}{c}
2 n \\
n+1
\end{array}\right)-\frac{1}{x}\left(\begin{array}{c}
2 n \\
n
\end{array}\right) \\
= & \frac{(x+1)^{2}}{x} P_{n}(x)+\frac{x-1}{x}\left(\begin{array}{c}
2 n \\
n
\end{array}\right)-\frac{1}{n+1}\left(\begin{array}{c}
2 n \\
n
\end{array}\right) .
\end{aligned}
$$

Proposition 32. The generating function $Q$ of the polynomials $P_{n}$ cf. (53) is given by

$$
Q(x, y)=\frac{y(x-1)}{\left(x-y(x+1)^{2}\right) \sqrt{1-4 y}}+\frac{x(\sqrt{1-4 y}+1)}{2\left(x-y(x+1)^{2}\right)} .
$$

The function $Q$ obeys

$$
\frac{\partial^{k+n} Q}{\partial x^{k} \partial y^{n}}(1,0)=n ! P_{n}^{(k)}(1)
$$


such that an explicit description for $Q$ allows us to calculate all $B(n, k)$ which then proves lemma 16 .

Proof. We define

$$
Q(x, y):=\sum_{n \geqslant 0} P_{n}(x) y^{n}
$$

and use the above recursion formula for $P_{n}$ as well as

$$
(1-4 y)^{-\frac{1}{2}}=\sum_{m \geqslant 0} \frac{\frac{1}{2}\left(\frac{1}{2}+1\right) \ldots\left(\frac{1}{2}+m-1\right)}{m !}(4 y)^{m}=\sum_{m \geqslant 0}\left(\begin{array}{c}
2 m \\
m
\end{array}\right) y^{m},
$$

and $\int(1-4 y)^{-\frac{1}{2}}=-\frac{1}{2}(1-4 y)^{\frac{1}{2}}$ which yields

$$
\begin{aligned}
Q(x, y)= & 1+\sum_{n \geqslant 1} P_{n}(x) y^{n}=1+y \sum_{n \geqslant 0} P_{n+1}(x) y^{n} \\
= & 1+\frac{y(x+1)^{2}}{x} \sum_{n \geqslant 0} P_{n}(x) y^{n}+\frac{y(x-1)}{x} \sum_{n \geqslant 0}\left(\begin{array}{c}
2 n \\
n
\end{array}\right) y^{n} \\
& -y \sum_{n \geqslant 0} \frac{1}{n+1}\left(\begin{array}{c}
2 n \\
n
\end{array}\right) y^{n} \\
= & \frac{y(x+1)^{2}}{x} Q(x, y) \\
& +1+\frac{y(x-1)}{x} \sum_{n \geqslant 0}\left(\begin{array}{c}
2 n \\
n
\end{array}\right) y^{n}-\int_{0}^{y} \sum_{n \geqslant 0}\left(\begin{array}{c}
2 n \\
n
\end{array}\right) t^{n} d t \\
= & \frac{y(x+1)^{2}}{x} Q(x, y)+1+\frac{y(x-1)}{x \sqrt{1-4 y}}+\frac{1}{2} \sqrt{1-4 y}-\frac{1}{2},
\end{aligned}
$$

from which we get (55).

The derivatives of $Q$ with respect to $x$ at the point $(1, y)$ are

$$
\begin{aligned}
Q(1, y) & =\frac{1}{2}(1-4 y)^{-\frac{1}{2}}+\frac{1}{2}(1-4 y)^{-1} \\
\frac{\partial Q}{\partial x}(1, y) & =y(1-4 y)^{-\frac{3}{2}} \\
\frac{\partial^{2} Q}{\partial x^{2}}(1, y) & =-y(1-4 y)^{-\frac{3}{2}}+y(1-4 y)^{-2} \\
\frac{\partial^{3} Q}{\partial x^{3}}(1, y) & =3 y(1-4 y)^{-\frac{3}{2}}+6 y^{2}(1-4 y)^{-\frac{5}{2}}-3 y(1-4 y)^{-2} \\
\frac{\partial^{4} Q}{\partial x^{4}}(1, y) & =-3 y(1-4 y)^{-\frac{3}{2}}-9 y(1-4 y)^{-\frac{5}{2}}+9 y(1-4 y)^{-2}+3 y(1-4 y)^{-3}, \\
\frac{\partial^{5} Q}{\partial x^{5}}(1, y) & =120 y^{3}(1-4 y)^{-\frac{7}{2}}-60 y(1-4 y)^{-2}+60 y(1-4 y)^{-\frac{5}{2}}-120 y^{2}(1-4 y)^{-3} .
\end{aligned}
$$


All terms are of the form $y^{m}(1-4 y)^{-\ell}$ with $m=0,1,2,3$ and the needed derivatives are given by

$$
\left.\frac{d^{n}}{d y^{n}}(1-4 y)^{-\ell}\right|_{y=0}= \begin{cases}2^{2 n} \frac{(\ell+n-1) !}{(\ell-1) !}, & \ell \in \mathbb{N} \\ \frac{(2 n+2 \ell-1) !\left(\ell-\frac{1}{2}\right) !}{\left(n+\ell-\frac{1}{2}\right) !(2 \ell-1) !}, & \ell \in \mathbb{N}+\frac{1}{2}\end{cases}
$$

as well as

$$
\left.\frac{d^{n}}{d y^{n}}\left(y^{k}(1-4 y)^{-\ell}\right)\right|_{y=0}= \begin{cases}\left.\left(\begin{array}{l}
n \\
k
\end{array}\right) k ! \frac{d^{n-k}}{d y^{n-k}}(1-4 y)^{-\ell}\right|_{y=0} & k \leqslant n \\
0 & \text { else }\end{cases}
$$

Therefore the partial derivatives of $Q$ with respect to $y$ at $(1,0)$ are

$$
\begin{aligned}
\frac{\partial^{n} Q}{\partial y^{n}}(1,0) & =2^{2 n-1} n !+2^{-1} \frac{(2 n) !}{n !} \\
\frac{\partial^{n+1} Q}{\partial y^{n} \partial x}(1,0) & =2^{-1} \frac{(2 n) !}{n !} n \\
\frac{\partial^{n+2} Q}{\partial y^{n} \partial x^{2}}(1,0) & =-2^{-1} \frac{(2 n) !}{n !} n+2^{2 n-2} n ! n \\
\frac{\partial^{n+3} Q}{\partial y^{n} \partial x^{3}}(1,0) & =2^{-1} \frac{(2 n) !}{n !} n(n+2)-3 \cdot 2^{2 n-2} n ! n \\
\frac{\partial^{n+4} Q}{\partial y^{n} \partial x^{4}}(1,0) & =-3 \frac{(2 n) !}{n !} n(n+1)+3 \cdot 2^{2 n-3} n ! n(n+7) \\
\frac{\partial^{n+5} Q}{\partial y^{n} \partial x^{5}}(1,0) & =\frac{(2 n) !}{n !} n\left(n^{2}+17 n+12\right)-15 \cdot 2^{2 n-2} n ! n(n+3) .
\end{aligned}
$$

From this we get with (54) and (57):

$$
\begin{aligned}
B(n, 0) & =2^{2 n-1}+\frac{1}{2}\left(\begin{array}{c}
2 n \\
n
\end{array}\right), & B(n, 1) & =\frac{1}{2}\left(\begin{array}{c}
2 n \\
n
\end{array}\right) n \\
B(n, 2) & =2^{2 n-2} n, & B(n, 3) & =\frac{1}{2}\left(\begin{array}{c}
2 n \\
n
\end{array}\right) n^{2}, \\
B(n, 4) & =2^{2 n-3} n(3 n-1), & B(n, 5) & =\frac{1}{2}\left(\begin{array}{c}
2 n \\
n
\end{array}\right) n^{2}(2 n-1),
\end{aligned}
$$

which - last but not least - yields lemma 16,

A variant of (52) is used in the proof of theorem 19, We define

$$
\widetilde{B}(n, k):=\sum_{j=0}^{n} j^{k}\left(\begin{array}{c}
2 n-1 \\
n-j
\end{array}\right) .
$$

Then usual manipulation of the binomials yields the following lemma

Lemma 33. The sums $B(n, k)$ and $\widetilde{B}(n, k)$ are connected via

$$
2 n \widetilde{B}(n, k)=n B(n, k)+B(n, k+1) .
$$




\section{Appendix B. Calculations for the proof of (40).}

For the proof of (40) we expand the left hand side and notice that we can extend the sums for $b$, because the added summands cancel or vanish. This yields

$$
\begin{aligned}
& 6(2 k+1) \sum_{0 \leqslant q \leqslant p \leqslant k} \operatorname{dim} V_{\left(2^{k-p}, 1^{p-q}, 0^{q}\right)}^{\mathfrak{s p}(2 k)} \operatorname{dim} V_{(p, q)}^{\mathfrak{s p}(4)} \\
& =\sum_{p=0}^{k} \sum_{q=0}^{p}(p-q+1)^{2}(p+q+3)(p+2)(q+1)\left(\begin{array}{c}
2 k+1 \\
k-q+1
\end{array}\right)\left(\begin{array}{c}
2 k+1 \\
k-p
\end{array}\right) \\
& -\sum_{p=0}^{k} \sum_{q=0}^{p}(p-q+1)^{2}(p+q+3)(p+2)(q+1)\left(\begin{array}{c}
2 k+1 \\
k-q-2
\end{array}\right)\left(\begin{array}{c}
2 k+1 \\
k-p-3
\end{array}\right) \\
& +\sum_{p=0}^{k} \sum_{q=0}^{p} \sum_{b=1}^{3}(p-q+1)(p-q-3+2 b)(p+q+3)(p+2)(q+1) . \\
& \cdot\left(\begin{array}{c}
2 k+1 \\
k-q+b-3
\end{array}\right)\left(\begin{array}{c}
2 k+1 \\
k-p-b
\end{array}\right) \\
& -\sum_{p=0}^{k} \sum_{q=0}^{p} \sum_{b=0}^{2}(p-q+1)(p-q-1+2 b)(p+q+3)(p+2)(q+1) . \\
& \cdot\left(\begin{array}{c}
2 k+1 \\
k-q+b-1
\end{array}\right)\left(\begin{array}{c}
2 k+1 \\
k-p-b
\end{array}\right) \\
& =\sum_{p=0}^{k} \sum_{q=0}^{p}(p-q+1)^{2}(p+q+3)(p+2)(q+1)\left(\begin{array}{c}
2 k+1 \\
k-q+1
\end{array}\right)\left(\begin{array}{c}
2 k+1 \\
k-p
\end{array}\right) \\
& -\sum_{p=0}^{k} \sum_{q=0}^{p}(p-q+1)^{2}(p+q+3)(p+2)(q+1)\left(\begin{array}{c}
2 k+1 \\
k-q-2
\end{array}\right)\left(\begin{array}{c}
2 k+1 \\
k-p-3
\end{array}\right) \\
& +\sum_{p=0}^{k} \sum_{q=0}^{p} \sum_{b=0}^{2}(p-q+1)(p-q-1+2 b)(p+q+3)(p+2)(q+1) . \\
& \cdot\left(\begin{array}{c}
2 k+1 \\
k-q+b-2
\end{array}\right)\left(\begin{array}{c}
2 k+1 \\
k-p-b-1
\end{array}\right) \\
& -\sum_{p=0}^{k} \sum_{q=0}^{p} \sum_{b=0}^{2}(p-q+1)(p-q-1+2 b)(p+q+3)(p+2)(q+1) \text {. } \\
& \cdot\left(\begin{array}{c}
2 k+1 \\
k-q+b-1
\end{array}\right)\left(\begin{array}{c}
2 k+1 \\
k-p-b
\end{array}\right) .
\end{aligned}
$$

With

$$
\left(\begin{array}{c}
n \\
m
\end{array}\right)\left(\begin{array}{c}
n \\
\ell
\end{array}\right)-\left(\begin{array}{c}
n \\
m-1
\end{array}\right)\left(\begin{array}{c}
n \\
\ell-1
\end{array}\right)=\frac{n-m-\ell+1}{n+1}\left(\begin{array}{c}
n+1 \\
m
\end{array}\right)\left(\begin{array}{c}
n+1 \\
\ell
\end{array}\right)
$$

we get

$$
=\sum_{p=0}^{k} \sum_{q=0}^{p}(p-q+1)^{2}(p+q+3)(p+2)(q+1)\left(\begin{array}{c}
2 k+1 \\
k-q+1
\end{array}\right)\left(\begin{array}{c}
2 k+1 \\
k-p
\end{array}\right)
$$


THE DECOMPOSITION OF THE SPINOR BUNDLE OF GRASSMANN MANIFOLDS

$$
\begin{aligned}
& -\sum_{p=0}^{k} \sum_{q=0}^{p}(p-q+1)^{2}(p+q+3)(p+2)(q+1)\left(\begin{array}{c}
2 k+1 \\
k-q-2
\end{array}\right)\left(\begin{array}{c}
2 k+1 \\
k-p-3
\end{array}\right) \\
& -\sum_{p=0}^{k} \sum_{q=0}^{p} \sum_{b=0}^{2} \frac{(p-q+1)(p-q-1+2 b)(p+q+3)^{2}(p+2)(q+1)}{2 k+2} . \\
& \cdot\left(\begin{array}{c}
2 k+2 \\
k-q+b-1
\end{array}\right)\left(\begin{array}{c}
2 k+2 \\
k-p-b
\end{array}\right) \\
= & \sum_{p=0}^{k+1} \sum_{q=0}^{p}(p-q)^{2}(p+q+2)(p+1)(q+1)\left(\begin{array}{c}
2 k+1 \\
k-q+1
\end{array}\right)\left(\begin{array}{c}
2 k+1 \\
k-p+1
\end{array}\right) \\
& -\sum_{p=0}^{k+1} \sum_{q=0}^{p}(p-q)^{2}(p+q+2)(p+1)(q+1)\left(\begin{array}{c}
2 k+1 \\
k-q-2
\end{array}\right)\left(\begin{array}{c}
2 k+1 \\
k-p-2
\end{array}\right) \\
& -\sum_{p=1}^{k+1} \sum_{q=1}^{p+2} \frac{(p-q+2)(p-q)(p+q)^{2}(p+1)(q-1)}{2 k+2}\left(\begin{array}{c}
2 k+2 \\
k-q+1
\end{array}\right)\left(\begin{array}{c}
2 k+2 \\
k-p+1
\end{array}\right) \\
& -\sum_{p=2}^{k+1} \sum_{q=0}^{p} \frac{(p-q)^{2}(p+q)^{2} p q}{2 k+2}\left(\begin{array}{c}
2 k+2 \\
k-q+1
\end{array}\right)\left(\begin{array}{c}
2 k+2 \\
k-p+1
\end{array}\right) \\
& -\sum_{q=3}^{k+1} \sum_{p=0}^{q-2} \frac{(p-q+2)(p-q)(q+p)^{2}(q-1)(p+1)}{2 k+2}\left(\begin{array}{c}
2 k+2 \\
k-q+1
\end{array}\right)\left(\begin{array}{c}
2 k+2 \\
k-p+1
\end{array}\right) .
\end{aligned}
$$

We use the symmetry of the summands with respect to $p$ and $q$ so that we arrive at

$$
\begin{aligned}
= & \frac{1}{2} \sum_{p=0}^{k+1} \sum_{q=0}^{k+1}(p-q)^{2}(p+q+2)(p+1)(q+1)\left(\begin{array}{c}
2 k+1 \\
k-q+1
\end{array}\right)\left(\begin{array}{c}
2 k+1 \\
k-p+1
\end{array}\right) \\
& -\frac{1}{2} \sum_{p=0}^{k+1} \sum_{q=0}^{k+1}(p-q)^{2}(p+q-4)(p-2)(q-2)\left(\begin{array}{c}
2 k+1 \\
k-q+1
\end{array}\right)\left(\begin{array}{c}
2 k+1 \\
k-p+1
\end{array}\right) \\
& -\sum_{p=0}^{k+1} \sum_{q=0}^{k+1} \frac{(p-q+2)(p-q)(p+q)^{2}(p+1)(q-1)}{2(k+1)}\left(\begin{array}{c}
2 k+2 \\
k-q+1
\end{array}\right)\left(\begin{array}{c}
2 k+2 \\
k-p+1
\end{array}\right) \\
& -\sum_{p=0}^{k+1} \sum_{q=0}^{k+1} \frac{(p-q)^{2}(p+q)^{2} p q}{4(k+1)}\left(\begin{array}{c}
2 k+2 \\
k-q+1
\end{array}\right)\left(\begin{array}{c}
2 k+2 \\
k-p+1
\end{array}\right) \\
& -\sum_{p=0}^{k+1} \frac{p^{3}(p+1)(p+2)}{2(k+1)}\left(\begin{array}{c}
2 k+2 \\
k-p+1
\end{array}\right)\left(\begin{array}{c}
2 k+2 \\
k+1
\end{array}\right) \\
& -2 \sum_{p=0}^{k+1} p^{2}(p-4)(p-2)\left(\begin{array}{c}
2 k+1 \\
k+1
\end{array}\right)\left(\begin{array}{c}
2 k+1 \\
k-p+1
\end{array}\right) \\
& -\sum_{p=0}^{k+1}(p-1)^{2}(p-3)(p-2)\left(\begin{array}{c}
2 k+1 \\
k
\end{array}\right)\left(\begin{array}{c}
2 k+1 \\
k-p+1
\end{array}\right) \\
& +6\left(\begin{array}{c}
2 k+1 \\
k
\end{array}\right)\left(\begin{array}{c}
2 k+1 \\
k+1
\end{array}\right)
\end{aligned}
$$




$$
\begin{aligned}
= & \frac{1}{2} \sum_{p=0}^{k+1} \sum_{q=0}^{k+1}(p-q)^{2}((p+q+2)(p+1)(q+1)-(p+q-4)(p-2)(q-2)) . \\
& \cdot\left(\begin{array}{c}
2 k+1 \\
k-q+1
\end{array}\right)\left(\begin{array}{c}
2 k+1 \\
k-p+1
\end{array}\right) \\
& -\frac{1}{4(k+1)} \sum_{p=0}^{k+1} \sum_{q=0}^{k+1}(p+q)^{2}(p-q)(2(p-q+2)(p+1)(q-1)+(p-q) p q) . \\
& \cdot\left(\begin{array}{c}
2 k+2 \\
k-q+1
\end{array}\right)\left(\begin{array}{c}
2 k+2 \\
k-p+1
\end{array}\right) \\
& \frac{1}{2(k+1)}\left(\begin{array}{c}
2 k+2 \\
k+1
\end{array}\right) \sum_{p=0}^{k+1} p^{3}(p+1)(p+2)\left(\begin{array}{c}
2 k+2 \\
k-p+1
\end{array}\right) \\
& -\left(\begin{array}{c}
2 k+1 \\
k
\end{array}\right) \sum_{p=0}^{k+1}(p-2)\left(2 p^{2}(p-4)+(p-1)^{2}(p-3)\left(\begin{array}{c}
2 k+1 \\
k-p+1
\end{array}\right) .\right. \\
& +6\left(\begin{array}{c}
2 k+1 \\
k
\end{array}\right)^{2}
\end{aligned}
$$

We expand the polynomial coefficients and write the last sum in Terms of $\widetilde{B}$ and $B$ :

$$
\begin{aligned}
= & \widetilde{B}(k+1,4) \widetilde{B}(k+1,0)+6 \widetilde{B}(k+1,3) \widetilde{B}(k+1,1)-9 \widetilde{B}(k+1,2)^{2} \\
& -9 \widetilde{B}(k+1,3) \widetilde{B}(k+1,0)+9 \widetilde{B}(k+1,2) \widetilde{B}(k+1,1) \\
& +18 \widetilde{B}(k+1,2) \widetilde{B}(k+1,0)-18 \widetilde{B}(k+1,1)^{2} \\
& -\frac{1}{2(k+1)}\left(3 B(k+1,5) B(k+1,1)-6 B(k+1,4) B(k+1,0)-3 B(k+1,3)^{2}\right. \\
& \left.+6 B(k+1,2)^{2}+\left(\begin{array}{c}
2 k+2 \\
k+1
\end{array}\right)(B(k+1,5)+3 B(k+1,4)+2 B(k+1,3))\right) \\
& -\left(\begin{array}{c}
2 k+1 \\
k
\end{array}\right)(2 \widetilde{B}(k+1,4)-12 \widetilde{B}(k+1,3)+16 \widetilde{B}(k+1,2)+\widetilde{B}(k+1,4) \\
& -7 \widetilde{B}(k+1,3)+17 \widetilde{B}(k+1,2)-17 \widetilde{B}(k+1,1)+6 \widetilde{B}(k+1,0)) \\
& +6\left(\begin{array}{c}
2 k+1 \\
k
\end{array}\right)\left(\begin{array}{c}
2 k+1 \\
k+1
\end{array}\right) .
\end{aligned}
$$

Inserting (60) as well as (58) from appendix $\mathrm{A}$ yields (40) which finishes the proof of theorem 19.

\section{REFERENCES}

[1] Arthur L. Besse. Einstein manifolds, volume 10 of Ergebnisse der Mathematik und ihrer Grenzgebiete (3) [Results in Mathematics and Related Areas (3)]. Springer-Verlag, Berlin, 1987.

[2] M. Cahen and S. Gutt. Spin structures on compact simply connected Riemannian symmetric spaces. In Proceedings of the Workshop on Clifford Algebra, Clifford Analysis and their Applications in Mathematical Physics (Ghent, 1988), volume 62, pages 209-242, 1988. 
[3] Roberto Camporesi and Emmanuel Pedon. The continuous spectrum of the Dirac operator on noncompact Riemannian symmetric spaces of rank one. Proc. Amer. Math. Soc., 130(2):507516 (electronic), 2002.

[4] J. A. Castilho Alcarás, J. Tambergs, T. Krasta, J. Ruža, and O. Katkevičius. Plethysms and interacting boson models. J. Math. Phys., 44(11):5296-5319, 2003.

[5] N. El Samra and R. C. King. Dimensions of irreducible representations of the classical Lie groups. J. Phys. A, 12(12):2317-2328, 1979.

[6] H.D. Fegan and B. Steer. First order differential operators on a locally symmetric space. Pac. J. Math., 194(1):83-96, 2000.

[7] Thomas Friedrich and Stefan Ivanov. Parallel spinors and connections with skew-symmetric torsion in string theory. Asian J. Math., 6(2):303-335, 2002.

[8] William Fulton and Joe Harris. Representation theory, volume 129 of Graduate Texts in Mathematics. Springer-Verlag, New York, 1991. A first course, Readings in Mathematics.

[9] S. Goette. Equivariant $\eta$-invariants of homogeneous spaces. (Äquivariante $\eta$-Invarianten homogener Räume.) (German). Shaker Verlag, 1997.

[10] S. Goette and U. Semmelmann. The point spectrum of the Dirac operator on noncompact symmetric spaces. Proc. Amer. Math. Soc., 130(3):915-923 (electronic), 2002.

[11] Sigurdur Helgason. Differential geometry, Lie groups, and symmetric spaces, volume 34 of Graduate Studies in Mathematics. American Mathematical Society, Providence, RI, 2001. Corrected reprint of the 1978 original.

[12] Roger Howe, Eng-Chye Tan, and Jeb F. Willenbring. Stable branching rules for classical symmetric pairs. Trans. Am. Math. Soc., 357(4):1601-1626, 2005.

[13] James E. Humphreys. Introduction to Lie algebras and representation theory. 3rd printing, rev. Graduate Texts in Mathematics, 9. New York - Heidelberg - Berlin: Springer-Verlag. XII, 171 p. DM 42.00; \$24.80, 1980.

[14] R. C. King and B. G. Wybourne. Some noteworthy spin plethysms. J. Phys. A, 15(4):11371141, 1982.

[15] Kazuhiko Koike and Itaru Terada. On the decomposition of tensor products of representations of the classical groups: by means of the universal character. Adv. Math., 74:57-86, 1989.

[16] Kazuhiko Koike and Itaru Terada. Young-diagrammatic methods for the representation theory of the classical groups of type $B_{n}, C_{n}, D_{n} . J$. Algebra, 107(2):466-511, 1987.

[17] Kazuhiko Koike and Itaru Terada. Young diagrammatic methods for the restriction of representations of complex classical groups to reductive groups of maximal rank. Adv. Math., 79:104-135, 1990

[18] Dudley E. Littlewood. The Theory of Group Characters and Matrix Representations of Groups. Oxford University Press, New York, 1940.

[19] V. D. Lyakhovskiı̌ and A. I. Mudrov. Spinor structures on homogeneous spaces. Teoret. Mat. Fiz., 92(1):13-23, 1992.

[20] I.G. Macdonald. Symmetric functions and Hall polynomials. 2nd ed. Oxford Science Publications. Oxford: Clarendon Press. x, 475 p. , 1998.

[21] W. G. McKay and J. Patera. Tables of dimensions, indices, and branching rules for representations of simple Lie algebras, volume 69 of Lecture Notes in Pure and Applied Mathematics. Marcel Dekker Inc., New York, 1981.

[22] Jean-Louis Milhorat. Spectrum of the Dirac operator on $\operatorname{Gr}_{2}\left(\mathbf{C}^{m+2}\right)$. J. Math. Phys., 39(1):594-609, 1998.

[23] Jean-Louis Milhorat. The first eigenvalue of the Dirac operator on compact Spin symmetric spaces. Comm. Math. Phys., 259(1):71-78, 2005.

[24] Jean-Louis Milhorat. A formula for the first eigenvalue of the Dirac operator on compact spin symmetric spaces. J. Math. Phys., 47(4):043503, 12, 2006.

[25] R. Parthasarathy. Dirac operator and the discrete series. Ann. of Math. (2), 96:1-30, 1972.

[26] L. Seeger. The spectrum of the Dirac operator on $G_{2} / \mathrm{SO}(4)$. Ann. Global Anal. Geom., 17(4):385-396, 1999.

[27] Hartmut Strese. Über den Dirac-Operator auf Grassmann-Mannigfaltigkeiten. Math. Nachr., 98:53-59, 1980.

[28] C. Tsukamoto. Spectra of Laplace-Beltrami operators on $\mathrm{SO}(n+2) / \mathrm{SO}(2) \times \mathrm{SO}(n)$ and $\operatorname{Sp}(n+1) / \operatorname{Sp}(1) \times \operatorname{Sp}(n)$. Osaka J. Math., 18:407-426, 1981.

[29] D. V. Vasilevich and V. D. Lyakhovskiı̌. The method of special imbeddings for grand unification models. Teoret. Mat. Fiz., 66(3):350-359, 1986. 
[30] Joseph A. Wolf. Complex homogeneous contact manifolds and quaternionic symmetric spaces. J. Math. Mech., 14:1033-1047, 1965.

[31] Brian G. Wybourne. Symmetry Principles and Atomic Spectroscopy. Wiley-Interscience [A division of John Wiley \& Sons], New York-London-Sydney, 1970.

Department of Mathematics, University of Dortmund, D-44221 Dortmund

FRANK.KLINKER@MATHEMATIK.UNI-DORTMUND.DE 